Draft VERSion MAY 28, 2018

Preprint typeset using LATEX style emulateapj v. 08/22/09

\title{
PROBING SPECTROSCOPIC VARIABILITY OF GALAXIES \& NARROW-LINE ACTIVE GALACTIC NUCLEI IN THE SLOAN DIGITAL SKY SURVEY
}

\author{
C. W. Yip ${ }^{1}$, A. J. Connolly ${ }^{2}$, D. E. Vanden Berk ${ }^{3}$, R. Scranton ${ }^{4}$, S. Krughoff $^{2}$, A. S. Szalay ${ }^{1}$, L. Dobos $^{5}$, \\ C. Tremonti ${ }^{6}$, M. Taghizadeh-Popp ${ }^{1}$, T. Budavári ${ }^{1}$, I. Csabai $^{5}$, R. F. G. Wyse ${ }^{1}$, Ž. Ivezići ${ }^{2}$ \\ Draft version May 28, 2018
}

\begin{abstract}
Under the unified model for active galactic nuclei (AGNs), narrow-line (Type 2) AGNs are, in fact, broad-line (Type 1) AGNs but each with a heavily obscured accretion disk. We would therefore expect the optical continuum emission from Type 2 AGN to be composed mainly of stellar light and non-variable on the time-scales of months to years. In this work we probe the spectroscopic variability of galaxies and narrow-line AGNs using the multi-epoch data in the Sloan Digital Sky Survey (SDSS) Data Release 6. The sample contains 18,435 sources for which there exist pairs of spectroscopic observations (with a maximum separation in time of $\sim 700$ days) covering a wavelength range of $3900-8900 \AA$ A. To obtain a reliable repeatability measurement between each spectral pair, we consider a number of techniques for spectrophotometric calibration resulting in an improved spectrophotometric calibration of a factor of two. From these data we find no obvious continuum and emission-line variability in the narrow-line AGNs on average - the spectroscopic variability of the continuum is $0.07 \pm 0.26 \mathrm{mag}$ in the $g$ band and, for the emission-line ratios $\log _{10}([\mathrm{~N} \mathrm{II}] / \mathrm{H} \alpha)$ and $\log _{10}([\mathrm{O} \mathrm{III}] / \mathrm{H} \beta)$, the variability is $0.02 \pm 0.03$ dex and $0.06 \pm 0.08$ dex, respectively. From the continuum variability measurement we set an upper limit on the ratio between the flux of varying spectral component, presumably related to AGN activities, and that of host galaxy to be $\sim 30 \%$. We provide the corresponding upper limits for other spectral classes, including those from the BPT diagram, eClass galaxy classification, stars and quasars.
\end{abstract}

Subject headings: galaxies: general - techniques: spectroscopic

\section{INTRODUCTION}

Active galactic nuclei (AGNs) are found to vary in the time-domain at many frequencies. The underlying physical mechanism driving the variability seen in AGN spectra is unknown (e.g., Peterson 2001; Bono et al. 2003). Several scenarios have been proposed including accretion disk instability (Kawaguchi et al. 1998), supernova explosion (Aretxaga et al. 1997), microlensing by stars in the intervening galaxy (Hawkins 1993), and varying ionizing source or/and varying optical depth of the material in the vicinity of light-generating regions (e.g., Tohline \& Osterbrock 1976; Goodrich 1989). At present, models involving non-thermal emission from the jets or events occurring on the AGN accretion disk are most favored (see, e.g., Stalin et al. 2004, for a summary of variability models in AGNs by radio loudness).

Most variability studies, however, have been focused on Type 1 (broad-line) AGNs. While the emission lines of Type 2 (narrow-line) AGNs were found to vary in the $\mathrm{X}$-rays on time-scales from hours to years (Guainazzi et al. 1998; Mueller et al. 2003; Mateos et al. 2007), their op-

\footnotetext{
${ }^{1}$ Department of Physics and Astronomy, The Johns Hopkins University, 3701 San Martin Drive, Baltimore, MD 21218, USA.

2 Astronomy Department, University of Washington, WA 98195, USA.

3 Saint Vincent College, 300 Fraser Purchase Road, Latrobe, PA 15650, USA.

${ }^{4}$ Department of Physics and Astronomy, University of Pittsburgh, 3941 O'Hara Street, Pittsburgh, Pennsylvania 15260, USA

${ }^{5}$ Department of Physics of Complex Systems, Eötvös Loránd University, H-1117 Budapest, Hungary.

${ }^{6}$ Steward Observatory, University of Arizona, 933 N. Cherry Avenue, Tucson AZ 85721, USA.
}

tical continua and emission lines are generally considered to be non-variable. For the emissions from the narrowline regions, the spatially low-density region (electron density $n_{e} \sim 1,000 \mathrm{~cm}^{-3}$ ) results in a long ( $\sim 100$ years $)$ recombination time $^{7}$ (Peterson et al. 1995), exceeding the duration of a typical observation. For the optical continuum, it is commonly considered to be composed mainly of the stellar light, in accord with the expectation from the unification model (Antonucci 1993; Urry \& Padovani 1995) in which Type 2 objects are obscured by a dusty torus along the line-of-sight. Dominated by the stellar light, the optical continuum in Type 2 AGNs should therefore be temporally non-variable. There is, however, no strong observational evidence in the timedomain to either support or dispute these ideas.

From a 4-year monitoring of the broad-band optical variability of 35 Type 1 and 2 Seyfert galaxies, Winkler et al. (1992) demonstrated that most galaxies in their sample were variable. Another promising work to probe Type 2 AGN variability in the optical is the intra-night variability of Seyfert 2 galaxies observed by Jang (2001), where two out of the three objects varied by $\sim 0.25 \mathrm{mag}$. Further, Trippe et al. (2008) found a Type 1.9 Seyfert galaxy (Osterbrock 1989) to change into a Type 2 Seyfert over a few years, and proposed the underlying cause to be a varying ionizing continuum.

The UV-optical variability of broad-line AGNs is bet-

\footnotetext{
7 The recombination time $\tau_{e}$ is referred to that of the hydrogen atom, so that $\tau_{e}=1 /\left(n_{e} \alpha_{A}\right)$, where $n_{e}$ is the electron density. The total recombination coefficient of hydrogen, $\alpha_{A}$, is equal to $4.18 \times 10^{-13} \mathrm{~cm}^{3} \mathrm{~s}^{-1}$ at an electronic temperature $T_{e}=10^{4} \mathrm{~K}$ (Osterbrock 1989).
} 
ter established with an amplitude of $\sim 10 \%$ (e.g., see discussions in Vanden Berk et al. 2004). Wavelength dependence of QSO variability has been studied by Wilhite et al. (2005), and the C IV-emission dependence by Wilhite et al. (2006) in the Sloan Digital Sky Survey (SDSS, York et al. 2000). In these studies the authors have developed a method to remove wavelength-dependent systematics which may exist in the spectra, from a spectroscopic plate $^{8}$ at one epoch of observation to the same plate at another epoch. Correcting for this on a plate-to-plate basis they were able to select variable QSOs and to demonstrate a power-law-like QSO difference spectrum; which in turn can be explained by the change in the accretion rate in thermal accretion disk (Pereyra et al. 2006). QSO photometric variability in the SDSS has been studied by Vanden Berk et al. (2004) for its dependency on physical parameters such as redshift, luminosity and radio properties; by de Vries et al. (2003) in which the authors used both the SDSS and the historical observations (up to $\sim 50$ years) and found the time-scale of the variability to be $\sim 2$ years; and by Ivezić et al. (2004) and Sesar et al. (2006) in which the authors used the SDSS repeated imagings in combination with the Palomar Observatory Sky Survey (POSS) data to probe long term QSO variability (up to $\sim 50$ years), and constrained its time scale to be $\sim 1$ year in the restframe.

Normal "inactive" galaxies (for our purpose defined to be galaxies that are not Type 1 or Type 2 AGNs, nor lowionization nuclear emission regions - (LINERS, Heckman 1980)) are not observed to be variable; and are generally not expected to be. For the continua of galaxies, the time scale of observation is negligible compared with that of stellar evolution (for solar-mass main sequence stars, lifetime $\approx 10^{10}$ years). For emission-line regions, that arise from low-density environments and H II regions, similar arguments can be made to those for narrow-line regions, with the understanding that the electron density is likely to be lower $\left(n_{e} \sim 1-1,000 \mathrm{~cm}^{-3}\right.$, Spitzer 1978).

The goal of this work is, therefore, to probe spectral variability of galaxies and narrow-line AGNs using the SDSS multi-epoch observations. The SDSS data have the advantage of large sample size with the same spectrophotometric reduction, which is critical to our work because we expect any variability present in galaxies to be small (compared with the amplitude of the QSO UV-optical variability, for example). Several techniques are used to refine the spectroscopic calibration, including those by Wilhite et al. (2005). The resultant galaxy variability measurement is compared with that of stars and QSOs, independently analyzed in this work.

In $\S 2$ we discuss the samples used. In $\S 3$ we present the variability measure. In $\S 4$ we describe the refinement of the spectroscopic calibration. In $\S 5$ we present the galaxy continuum variability, and the emission line variability of the variable candidates based on the continuum. In $\S 6$ we present results on variability analyses on stars and QSOs as sanity checks. In $\S 7$ we summarize the results and discuss future work.

\section{SAMPLES}

8 A spectroscopic plate in the SDSS contains a set of spectra which are observed simultaneously.
As part of the SDSS (York et al. 2000) spectra are observed with fibers of $3^{\prime \prime}$ diameter (corresponding to $0.18 \mathrm{~mm}$ at the focal plane for the $2.5 \mathrm{~m}, \mathrm{f} / 5$ telescope, Gunn et al. 2006). The spectral resolution $R$ is $\sim 1800$ in the observed frame $3800-9200 \AA$. All sources are selected (or "targeted") from an initial imaging survey using the SDSS camera as described in Gunn et al. (1998) with the filter response curves as described in Fukugita et al. (1996), and using the imaging processing pipeline of Lupton et al. (2001). The astrometric calibration is described in Pier et al. (2003). The photometric system and calibration are described in Fukugita et al. (1996), Hogg et al. (2001), Smith et al. (2002) and Ivezić et al. (2004), Tucker et al. (2006). The targeting strategy for the multi-object spectrograph is described in Blanton et al. (2003).

We select our samples from the Data Release 6 (DR6, Adelman-McCarthy et al. 2008) of the SDSS. The galaxy sample is constructed from the SDSS Main Galaxy sample (Strauss et al. 2002), by selecting spectra which are both spectroscopically classified as galaxies, and rejecting spectra in which the redshift measurements failed or have not been made. The spectral classification (star, galaxy, QSO) is highly confident, with $98 \%$ of all the DR6 spectra having consistent classification between the spectro1d pipeline (SubbaRao et al. 2002) and the specBS pipeline (Schlegel et al. in prep.). From this sample, pairs of spectral observations are identified by matching sources in both RA and DEC to within $1^{\prime \prime}$, and in redshift to within 0.01. Spectra at both epochs are required to have the same plate number but different date of observations, given in Modified Julian Date (MJD) by the SDSS. The selection results in 23,330 pairs. The subsequent sample selections (mainly for the purpose of increasing the signal-to-noise, $\mathrm{S} / \mathrm{N}$, of the spectra) will be described in $\S 4$ and $\S 5$. Table 1 lists the relevant spectroscopic plates and the corresponding number of galaxy spectra. The sample covers $\sim 3-700$ days in the observed frame.

The spectroscopically classified galaxies selected above do not contain any strong (equivalent width, EW $>10 \AA$, and $>3 \sigma$ detection in the height of the line) broad line (full width at half maximum, FWHM $>1000 \mathrm{~km} \mathrm{~s}^{-1}$ ) (SubbaRao et al. 2002). The cutoff is below the traditional line-width selection for broad lines (Type 1) galaxies, where the width of permitted line(s) $>2000 \mathrm{~km} \mathrm{~s}^{-1}$ (e.g., Komossa 2008). The sample therefore comprises galaxies of different eClass types (Connolly et al. 1995), and narrow-line AGNs. A potential contamination to our galaxy sample would be the narrow-line Seyfert 1 galaxies (Osterbrock \& Pogge 1985, and references therein), expected to offer a direct view of the active nuclei (Antonucci 1993; Urry \& Padovani 1995) and can be variable in the optical (Giannuzzo \& Stirpe 1996; Peterson et al. 2000). Each of these spectra shows a broad component in Balmer emission line with FWHM $<2000 \mathrm{~km} \mathrm{~s}^{-1}$, and relatively weak $[\mathrm{O}$ III $] \lambda 5008$. The reasons behind this potential contamination are twofold - firstly, the spectrold pipeline fits to each line a single Gaussian, that may underestimate the line width of the broad component in the case of a composite line which is made up of both the broad and narrow components, in particular when the broad one is relatively weak. Secondly, the 
FWHM of the broad component of both $\mathrm{H} \alpha$ and $\mathrm{H} \beta$ of a narrow-line Seyfert 1 can extend down to $\sim 500 \mathrm{~km} \mathrm{~s}^{-1}$ (Fig. 4 of Zhou et al. 2006), falling within the galaxy spectral classification in the SDSS. However, we expect the number contribution of these objects to the whole DR6 galaxy+QSO sample to be small, $\sim 0.5 \%$, with reference to Zhou et al. (2006) who used an upper limit of $2200 \mathrm{~km} \mathrm{~s}^{-1}$ in the broad component of $\mathrm{H} \alpha$ or $\mathrm{H} \beta$ emissions to search for narrow-line Seyfert 1 in the SDSS. Similarly, the Seyfert 1.8 and 1.9 (Osterbrock 1989), both of which show strong narrow components (relative to the broad ones, if available) in $\mathrm{H} \alpha$ and $\mathrm{H} \beta$, can be variable in the optical (Goodrich 1989), and may be present in our galaxy sample as well. We note that the current analysis does not provide further separation of those from our emission-line galaxies, that calls for fitting of double Gaussian to each Balmer line, for example. The number contribution of these types to the full galaxy sample is expected to be small, $\sim 0.02 \%$ (Wang \& Wei 2008). In this work, the fraction of the classified Seyfert $2+$ starforming + composite galaxies to the full sample of our galaxy spectral pairs is $\sim 14 \%$; and that of all the eClass types, $\sim 22 \%$ (calculated from Table 4 ).

When constructing the stellar and QSO samples, the spectral pairs are selected similarly to the case of galaxies, except that they are spectroscopically classified as star and QSO, respectively. The targeting selection for spectroscopic observation of QSOs in the SDSS is described in Richards et al. (2002). Each QSO spectrum classified (SubbaRao et al. 2002) has at least one strong line (see above for the galaxy classification) with FWHM $>1000 \mathrm{~km} \mathrm{~s}^{-1}$, or/and a Lyman alpha forest. Unlike the selection of the galaxies and the QSOs, no criterion is imposed on the quality of the redshift when selecting the stars. The upper bound of their distances is $c z=450 \mathrm{~km} \mathrm{~s}^{-1}$, or $z=0.0015$ (SubbaRao et al. 2002). The samples contain 9,100 stellar pairs and 3,205 QSO pairs $(0.08<z<3.51)$, respectively.

The spectra are de-reddened against Galactic extinction using the library written by Simon Krughoff ${ }^{9}$, which adopts the SFD dust maps (Schlegel et al. 1998) and the extinction curve by O'Donnell (1994). Following the SDSS convention the spectra are expressed in vacuum wavelengths. Flux densities $f_{\lambda}$ are expressed in $10^{-17}$ ergs s$^{-1} \mathrm{~cm}^{-2} \AA^{-1}$.

\section{VARIABILITY AMPLITUDE: $F_{\text {var }}$}

We adopt the dimensionless variability measure, $F_{\text {var }}$, for $N$-epoch repeated observations (e.g., RodriguezPascual et al. 1997; Peterson 2001). It is a fractional root-mean-squared variability amplitude defined as

$$
F_{\mathrm{var}}=\frac{\sqrt{\sigma^{2}-\delta^{2}}}{\langle f\rangle},
$$

where $\sigma^{2}$ is the variance of the flux, $\delta^{2}$ is the mean square uncertainty of the flux, and $\langle f\rangle$ is the mean flux

$$
\sigma^{2}=\frac{1}{N} \sum_{i=1}^{N}\left(f_{i}-\langle f\rangle\right)^{2},
$$

9 The library is available from the author upon request.

$$
\begin{gathered}
\delta^{2}=\frac{1}{N} \sum_{i=1}^{N} \delta_{i}^{2}, \\
\langle f\rangle=\frac{1}{N} \sum_{i=1}^{N} f_{i} .
\end{gathered}
$$

The numerator, proposed by Bonoli et al. (1979), was termed the square-root of the "excess variance" (Vaughan et al. 2003), in the sense that the flux uncertainty is subtracted in quadrature from the variability. In the calculation of the continuum $F_{\text {var }}$ the wavelength intervals encompassing emission-line center wavelengths $\pm 280 \mathrm{~km} \mathrm{~s}^{-1}$ are excluded (using the vacuum wavelength values in the line list ${ }^{10}$ adopted by the SDSS, and excluding the lines $\mathrm{Ca} \mathrm{K}$ and $\mathrm{H}, \mathrm{H} \delta$, the $\mathrm{G}$ band around $4306 \AA, \operatorname{Mg} \lambda 5177, \mathrm{Na} \lambda 5896$, and the Ca II triplet that usually appear as absorptions). The mean square uncertainty for the spectrum at each epoch $\left(\delta_{i}^{2}\right.$ for a given $i$ ) is calculated by quadrature summation of the pipeline flux uncertainty per pixel, over all valid pixels. It is therefore a variance in flux based on photon statistics. A valid pixel is defined to be a pixel that is not flagged as bad. The flags are listed in $\S 4.2 . F_{\text {var }}$ is calculated in the observed frame with wavelengths $3900-8900 \AA$ for each object and for all spectral types.

When calculating $F_{\mathrm{var}}$ for narrow-line AGNs, since any possible host-galaxy contribution is not explicitly subtracted from an observed spectrum, the full observed spectrum is used in calculating the denominator $\langle f\rangle$. As the numerator term "excess variance" does not contain the non-variable host-galaxy contribution, the resultant $F_{\text {var }}$ is therefore a lower limit on the AGN variability.

If the excess variance $\sigma^{2}-\delta^{2}<0$, we assume the variability is zero for the object, but continue to include it in the analysis. In this work, $N=2$. We note that any nonzero $F_{\text {var }}$ is referred to as a "variability" measurement, regardless of the origin being physically interesting or otherwise.

\section{REFINING SPECTROSCOPIC CALIBRATION}

The principal steps in the spectrophotometric calibration of each spectrum in the SDSS are: a wavelengthdependent calibration using the observed standard stars on the same plate; and the tying of the absolute flux scale to the observed PSF magnitudes of stars, on the same plate (Adelman-McCarthy et al. 2008). The resultant average uncertainty in the spectrophotometry for DR6 is $7 \%$ at observed frame wavelength $3800 \AA$ (AdelmanMcCarthy et al. 2008). As any variability in the sources from our sample is expected to be of low amplitude, the calibration of the pipeline spectra must be improved using the approaches described below.

\subsection{Wavelength-dependent correction}

We adopt the method developed by Wilhite et al. (2005) to remove systematics which may be present in each pair of galaxy spectra taken on separate MJDs. Flux-density corrections as a function of the observedframe wavelength in the range of $3900-8900 \AA$ are calculated by a linear fit (tied to the origin) between the

\footnotetext{
10 Stoughton et al. (2002).
} 
flux density $\left(f_{\lambda}\right)$ of all the objects at epoch 2 (the later epoch) and those at epoch 1 . This calculation results in a calibration spectrum for one plate. We adopt a slightly different methodology from Wilhite et al. (2005) in which stellar spectra in each plate were used. Our calibration spectrum per plate is calculated using all galaxies present in that plate, as we want to maximize the number of objects in generating the calibration spectrum, and avoid potential systematic effects when applying point source calibration to extended sources. The underlying assumption is that the majority of the galaxies observed in a given plate are non-variable in time across the two epochs. Even if the galaxies were in fact variable, the method is still applicable because the variable amplitude of several hundred galaxies is not expected to be in phase. An example calibration spectrum is plotted in Fig. 1. Typically the calibration spectra are smooth. To focus on low-order corrections to the galaxy spectra, and to remove noise in the calibration spectrum, we follow Wilhite et al. (2005) and set the final calibration spectrum per plate as its 5 th-order polynomial least-squares fit. By visual inspection this functional form works well in removing higher-order spurious spectral features.

When constructing the calibration spectrum we drop any plate in which there are less than 10 pairs of galaxy spectra. We then refine each and every spectrum present in a given plate by using the calibration spectrum, if available.

\subsection{Wavelength-independent correction}

After removing wavelength-dependent systematics, each spectral pair can, in principle, be subjected to a wavelength-independent normalization in their flux densities. In order to obtain an accurate variability measurement of each spectral pair, the total continuum fluxes of the two spectra at a chosen common wavelength region are set equal. Specifically, for each pair the galaxy spectrum at the epoch of lower $\mathrm{S} / \mathrm{N}$ is shifted in a wavelengthindependent fashion according to

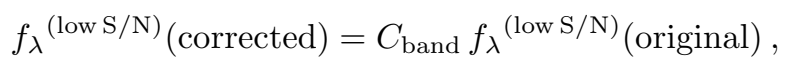

in which $C_{\text {band }}$ is a constant for each spectral pair, calculated as

$$
C_{\text {band }}=F_{\lambda \in \text { band }}(\text { high S } / \mathrm{N}) / F_{\lambda \in \text { band }}{ }^{(\text {low } \mathrm{S} / \mathrm{N})},
$$

where $F_{\lambda \in \text { band }}$ is the total flux in the restframe wavelength range (which is referred to as "rest-band" for convenience). In this work the rest-band is chosen to be $6450 \AA \pm 520 \mathrm{~km} \mathrm{~s}^{-1}$, as such no prominent emission lines are present in the galaxy spectra ${ }^{11}$. Only the galaxy spectra in the first epoch are re-calibrated because it is typically the existence of low $\mathrm{S} / \mathrm{N}$ data that results in a second observation. The combined procedure of systematics removal and restframe band scaling is referred to "SYS+BAND" in the following discussion.

Given an initial sample $(\S 2,23,330$ objects), we impose $\mathrm{S} / \mathrm{N}$ cuts using the rest-band measures (or the

11 When refining QSO spectra which are located at a wide range of redshift, an observed frame wavelength range $6450 \AA \pm 520 \mathrm{~km} \mathrm{~s}^{-1}$ is used.
[O III $] \lambda 5008$ (vacuum) line, described later when we discuss absolute flux calibration). In this case we reject any spectral pair if there are one or more pixels flagged in either or both spectra in the wavelength region of interest, and we require the flux to be larger than zero at both epochs. The flags under consideration are NOPLUG, BADTRACE, BADFLAT, BADARC, MANYBADCOL, MANYREJECT, LARGESHIFT, FULLREJECT, SCATTERLIGHT, CROSSTALK, NOSKY, BRIGHTSKY, NODATA, COMBINEREJ, BADFLUXFACTOR, BADSKYCHI and REDMONSTER. All of the spectral flux or $\mathrm{S} / \mathrm{N}$ calculations in this paper are carried out with pixel-masking using these flags. After this $\mathrm{S} / \mathrm{N}$ criteria, the number of objects in the galaxy sample is reduced to 18,435 (Table 1 ).

In Fig. 2 and 3 we show the restframe galaxy spectra in two epochs from the SDSS pipeline and after the calibration refinement, respectively. Firstly, we note that the pipeline performs well, that the fractional difference between both epoch is about $10 \%$ at the shortest wavelength, which is sufficient for many spectral analyses involving emission or absorption lines. However, continuum residual in Fig. 2 is present, which induces a variability $\left(F_{\text {var }}=0.058\right)$. After the calibration refinement we are able to obtain a zero continuum variability.

For comparing with the rest-band scaling previously discussed, we also adopt the absolute flux calibration described in Peterson et al. (1998). In this procedure, the spectrum in the low $\mathrm{S} / \mathrm{N}$ epoch in the duo is scaled wavelength-independently so that its total flux in [O III $] \lambda 5008$ (vacuum) is the same as that of the spectrum in the high $\mathrm{S} / \mathrm{N}$ epoch. The physical justification is that the $[\mathrm{O}$ III $] \lambda 5008$ line generally appears as a narrow line arising from a low-density region, which is believed to be non-variable in time (Peterson et al. 1995). We note that the average [O III] EW of galaxy spectra in the full DR6 catalog is a few $\AA$, so for some objects mainly the continuum flux is measured and is expected to be non-variable as well. The combined procedure of systematics removal and absolute [O III] calibration is called "SYS+OIII" in the following. The region of influence is set to be $5008 \AA \pm 280 \mathrm{~km} \mathrm{~s}^{-1}$.

When judging which method performs better in analyzing spectral variability, we consider the better method to be the one which minimizes the mean, one standard deviation of the mean $\left(\sigma_{m}\right)$ and the one standard deviation sample scatter $(\sigma)$ of the variability at a fixed spectral $\mathrm{S} / \mathrm{N}$. These statistics for the galaxy sample by using the SYS+BAND, SYS+OIII and other refinement methods are listed in Table 2. At $S / N \geq 10$ the $\mathrm{SYS}+\mathrm{BAND}$ method produces smaller mean and scatter in $F_{\text {var }}$. The difference is not related to the difference in the width of the rest-band being used $\left(520 \mathrm{~km} \mathrm{~s}^{-1}\right.$ vs $280 \mathrm{~km} \mathrm{~s}^{-1}$ ), as the SYS+BAND method is also reperformed using $6450 \AA \pm 280 \mathrm{~km} \mathrm{~s}^{-1}$, the same width as in the SYS+OIII method. These statistics remain lower in value than those in the SYS+OIII even for this reduced interval. The result suggests that the [O III $] \lambda 5008$ line in some spectra may be impacted by the effect of seeing so that the total observed line flux is different for the two epochs. The other possible reasons for this discrepancy are: the total line flux is sampled at only a few wavelength bins in the medium resolution SDSS spectra, 
or the line may exhibit intrinsic variability.

To further test the method SYS+BAND, we divide the galaxy sample into RED and BLUE galaxies by using the eClass classification (described in \$5.2.1). The RED spectra are defined as objects with classification parameter $\phi_{K L} \geq 0^{\circ}$, and the BLUE galaxies, $\phi_{K L}<0^{\circ}$. For each plate, the calibration spectrum is calculated using only the RED or BLUE galaxies, which in turn is used to re-calibrate galaxies of all spectral types on the same plate. The purpose behind this is to determine whether the refinement method SYS+BAND would be biased by the presence of potentially different galaxy spectral types in the plate. We find that using a specific spectral type does not cause the variability statistics to change (Table 2). We therefore adopt SYS+BAND as the calibration method, and use all available galaxy spectral types when calculating the calibration spectrum throughout this work. The "before and after" of this approach is shown in Fig. 4, where we see that the scatter in $F_{\text {var }}$ vs. $\mathrm{S} / \mathrm{N}$ of our galaxy sample is reduced after the refinement. Comparing the average variability of our galaxy sample using the SYS+BAND approach with that from the original SDSS spectral reductions (Table 2) we find that the spectrophotometry is improved by a factor of two.

\subsection{Plate-to-Plate Difference}

There are plate-to-plate differences seen in the above definitions of variable and non-variable sources, that is, the best-fit coefficients in Eqn. 7 are in general plate dependent. In Fig. 5 we show $\left\langle F_{\text {var }}\right\rangle$ of the galaxy spectra vs. average spectral S/N, per plate. The average $F_{\text {var }}$ per plate is larger at lower overall spectral $\mathrm{S} / \mathrm{N}$, suggesting in those plates the variability measurement is likely affected by the presence of noise in the spectra. By dropping individual spectral pairs with spectral $\mathrm{S} / \mathrm{N}<10$ at the second epoch, the average and the sample scatter in $F_{\text {var }}$ are reduced to 0.010 and 0.003 , respectively.

\section{GALAXY VARIABILITY}

\subsection{Defining Variables}

Following Wilhite et al. (2005) we define the variable candidates by objects having

$$
F_{\text {var }}(\mathrm{S} / \mathrm{N})>\mathrm{b}_{0} \exp \left(\frac{\mathrm{S} / \mathrm{N}}{\mathrm{b}_{1}}\right)+\mathrm{b}_{2} .
$$

The $\mathrm{S} / \mathrm{N}$ of each spectrum is calculated in the second epoch and by using all of the valid pixels as defined in $\S 4.2$. The constants $b_{0}, b_{1}, b_{2}$ are determined by least-squares fitting the above exponential form to the binned data $\left(\langle\mathrm{S} / \mathrm{N}\rangle,\left\langle F_{\mathrm{var}}\right\rangle+n \sigma\left(F_{\mathrm{var}}\right)\right)$, where $\sigma\left(F_{\mathrm{var}}\right)$ is one standard deviation of $F_{\text {var }}$ in each bin, and for $n=0,1,2,3,4$. Typically 10 bins in $\mathrm{S} / \mathrm{N}$ are used.

The variable candidates (for galaxies, stars and QSOs) in this work are defined as objects with $>3 \sigma$ detection. We do not sub-divide the distribution for detections greater than $4 \sigma$, though in the future we plan to explore in more detail the tail of the variable distributions. For comparison we have also defined variables as objects located in a fixed upper percentile of $F_{\mathrm{var}}$ in the plot $F_{\text {var }}$ vs. S/N. The $>3 \sigma$ cut is equivalent to a $2-6 \%$ upper percentile for a given S/N (Table 3).

\subsection{Continuum}

To explore any dependence of spectral variability or repeatability on the spectral type of galaxies, we divide the galaxy sample using two different spectral classification schemes: eClass (Connolly et al. 1995) and the "BPT" diagrams or the Osterbrock diagrams (Baldwin et al. 1981; Veilleux \& Osterbrock 1987). These two schemes are motivated by different spectral features. The eClass is a spectral type describing the steepness of the continuum slope and other higher-order spectral features by using a linear combination of several eigenspectra for each galaxy spectrum. The eClass parameters, $\phi_{K L}$ and $\theta_{K L}$, are related to the coefficients in the eigenspectra expansion (called eigencoefficients), which are calculated by the Spectro1d pipeline in the SDSS. Specifically, the angle $\phi_{K L}$ correlates with the $\mathrm{H} \alpha$ emission EW (Madgwick et al. 2003) which is an indicator of star formation rate. The angle $\theta_{K L}$ discriminates galaxy spectra exhibiting post-starburst activities (Connolly et al. 1995; Yip et al. 2004).

On the other hand, the BPT diagrams separate the narrow-line AGNs from galaxies such as the star-forming galaxies by emission line ratios which indicate the physical conditions of gas inside the galaxies such as the electron density and the mean level of ionization (Osterbrock 1989). The objects in these two spectral classification schemes are not mutually exclusive. We hereafter present results on variability within both kinds of classification.

\subsection{1. e Class}

The spectral variability as a function of $\mathrm{S} / \mathrm{N}$ per mean eClass galaxy type (Connolly et al. 1995) is shown in Table 4 , and Table 5 for only the variable candidates. The mean eClass spectra are galaxy spectra where each is averaged over a non-trivial size of subspace of the two eClass parameters $\phi_{K L}$ and $\theta_{K L}$ (Yip et al. 2004). Limited by the number of spectral pairs, $\leq 3$ in types $\mathrm{E}$ or $\mathrm{F}$, only the types A, B, C and D are considered in the variable candidates (Table 5). The continuum of the mean galaxy spectrum is reddest in the type $\mathrm{A}$ and bluest in the type D.

Firstly, we note that the average variability amplitude are consistent among different eClass types to within 1$2 \sigma$ sample scatter. If we assume a priori that some populations of galaxies do not vary in time, then a spectral repeatability can be assigned to the population with the smallest mean and scatter in $F_{\mathrm{var}}$, that is, the mean eClass type B. The spectral features of this class are similar to the elliptical galaxies in the atlas of nearby galaxies by Kennicutt (1992). The repeatability, taken to be the average $F_{\mathrm{var}}$, is 0.010 or $1.0 \%$ in the observed-frame wavelength range $3900-8900 \AA$.

\subsubsection{Emission-line galaxies: narrow-line AGN vs. star-forming}

Emission-line galaxies are considered by using the BPT diagram, using the starburst theoretical modeling line by Kewley et al. (2006). We consider the types: Seyfert 2, star-forming, composite and LINERs (Heckman 1980). We do not find any LINER in our galaxy spectral pairs, using the classification criteria by Kewley et al. (2006, their Eqn. 15) which involved [O I] $\lambda 6302$. We hereby consider the diagram $\log _{10}([\mathrm{O} \mathrm{III}] / \mathrm{H} \beta)$ vs. $\log _{10}([\mathrm{~N} \mathrm{II}] / \mathrm{H} \alpha)$ (Fig. 5 of Baldwin et al. 1981). 
In the first step of this analysis the EWs are taken from the SDSS reduction. Galaxies with any one of the four lines $(\mathrm{H} \beta$ at $4863 \AA$, [O III] $\lambda 5008, \mathrm{H} \alpha$ at $6565 \AA$, $[\mathrm{N}$ II] $]$ 6585) having restframe EW smaller than $1 \AA$ are rejected (in the SDSS convention EW $>0$ for emission lines); and at least a $2 \sigma$ detection is required in each of the four lines. Restframe stellar absorption of the related hydrogen emission lines in the BPT diagram is corrected for by a constant increment of $1.3 \AA$ (Hopkins et al. 2003; Miller et al. 2003). Any possible aperture effect on the emission lines is neglected in this paper, because the primary interest of this work is the comparison of line strength between two epochs, which is done using the same aperture for each object in each epoch and will only be subject to the difference in seeing. To correct on an object-by-object basis would require an assumption of an average line strength as a function of radius for each galaxy. Although the true line-flux correction of individual objects should depend on the distribution of the gas and its projected angle on the sky relative to the observational aperture, under the typical sky conditions in the SDSS observation (the median PSF width $=1.4^{\prime \prime}$ in the $r$ band, Fig. 4 of Abazajian et al. 2003) and given the diameter of the fiber, the seeing-induced aperture correction factor to $[\mathrm{O} \mathrm{III}]$ is close to unity, based on a nearby $(z=0.009)$ Seyfert 1 with a well-resolved $\left(\sim 10^{\prime \prime}\right)$ narrow-line region (Fig. 3 of Wanders et al. 1992). This means that seeing-induced differences in the [O III] flux between any two epochs should be small. Further, the SDSS $3^{\prime \prime}$ diameter fiber spectroscopy does not cause substantial aperture bias in classifying galaxy spectra based on emission lines (Miller et al. 2003).

In Table 4 we compare the variability amplitude between the star-forming galaxies and the narrow-line AGNs in the full galaxy sample, and Table 5 for the variable candidates. It is a little surprising that the average variability amplitude at $\mathrm{S} / \mathrm{N} \geq 10$ is $1.2 \%$ for the star-forming galaxies, slightly higher than that of the narrow-line AGNs, $0.9 \%$. The difference is bigger than $1 \sigma_{m}$ (i.e., the measurement of the $F_{\text {var }}$ difference is reliable), but smaller than the $1 \sigma$ sample scatter, indicating the $F_{\text {var }}$ of both types are consistent with each other.

In Table 6 we show the average absolute AB magnitudes in $g, r$ and $i$ bands of variable candidates in the dim phase, and the sample-averaged magnitude difference between the dim and bright phases, per spectral type. The magnitudes are calculated for each spectrum, by convolving each restframe-shifted spectrum with the SDSS filter response curves for extended sources at an airmass of 1.3 (Fukugita et al. 1996), and in the AB filter system (Oke \& Gunn 1983). The average difference in the $g$ band magnitudes ranges from $0.07-0.10$ mag depending on the spectral type, but they are consistent among different spectral types to within $1 \sigma$ sample scatter of the magnitude difference, $\sigma(\Delta M)$, a measure of the sum of the sample scatters in both the distributions of $M(\operatorname{dim})$ and $M$ (bright). For the narrow-line AGNs, no obvious continuum variability is found (e.g., $0.07 \pm 0.26 \mathrm{mag}$ in the $g$ band).

We note that the distribution of $F_{\mathrm{var}}$ in the full sample is non-Gaussian. Using the $>3 \sigma$ cut for the variable candidates, for a Gaussian distribution one would expect the ratio between the number of variable candidates and that of the whole sample to be

$$
\frac{1-\operatorname{erf}(3 / \sqrt{2})}{1-\operatorname{erf}(-\infty)}
$$

or $0.13 \%$,where $\operatorname{erf}(z)$ is the error function, and $1-$ $\operatorname{erf}(z)$ is the complementary error function of the form $(2 / \sqrt{\pi}) \int_{z}^{\infty} e^{-t^{2}} d t$. From Table 3 , we see that typically at various $\mathrm{S} / \mathrm{N}$ the $F_{\text {var }}$ of the variable candidates span a longer tail $(1.53-3.44 \%)$.

\subsubsection{Structure Function of the Continuum}

The characteristic time scale of variability is usually studied by constructing the structure function $(S F$, e.g., Simonetti et al. (1985), Hughes et al. (1992); and references therein), the variability amplitude $F_{\text {var }}$ as a function of time lag. Although no obvious continuum variability is found in our sample of galaxies on average, the $S F$ is nonetheless constructed for the galaxy sample. Through the $S F$ analysis, an absence of a time scale would be a cross-check of the lack of variability, and the comparison between the $S F$ of stars and of QSOs $(\S 6)$ with that of the galaxies can be carried out.

The restframe $S F, F_{\text {var }}$ vs. $\Delta \tau_{\text {rest }}$, of all galaxies in the galaxy sample is illustrated in Fig. 6 , where $\Delta \tau_{\text {rest }}=$ $\Delta \tau /(1+z)$, and $z$ being the redshift of a given galaxy. The time lag $\Delta \tau$ is the difference between the two MJDs of observation, MJD(epoch 2)-MJD(epoch 1). No obvious time-scale is seen in the above restframe $S F \mathrm{~s}$ of galaxies for the $0-500$ days (restframe) sampled. A similar conclusion is obtained when the fractional change in flux $(|\Delta f / f|)$ is used instead of $F_{\text {var }}$ (Fig. 7), where $\Delta f$ is the difference in the flux between two epochs, and $f$ is the average flux over the two epochs.

An exponential function of the form

$$
F_{\text {var }}=a\left(1-b e^{-\Delta \tau_{\text {rest }} / \tau_{s}}\right)
$$

is fitted to each structure function, where $\tau_{s}$ is the characteristic variability time scale. Typically, a structure function where $b=1$ is considered (e.g., Bonoli et al. 1979; Trevese et al. 1994). Firstly we relax $b$ to accommodate the non-zero variability at zero time lag, obvious in Fig. 6. This can be attributed to the repeatability of the continuum variability measurement $(\S 5.2 .1)$ in eClass Type B, where the mean $F_{\text {var }}$ is 0.010 or $1.0 \%$. The bestfit $\tau_{s}$ for the galaxies is $\sim 10$ years. Uncertainty of this time scale is large $\left(\sim 2\right.$ years, with a reduced $\chi^{2}$ of 20$)$ because the duration of the observation is much shorter than the best-fit $\tau_{s}$.

We then consider a typical $b=1$ structure functional form at Eqn. 9. By assuming $F_{\text {var }}$ at the zero time lag $\left(F_{\mathrm{var}}(0)\right)$ (the noise) is uncorrelated with the intrinsic $F_{\text {var }}$ (the signal), one can obtain the intrinsic $F_{\text {var }}$ by subtracting $F_{\text {var }}(0)$ from the total $F_{\text {var }}$ in a quadrature fashion. The resultant structure function is shown in Fig. 8, and the best-fit variability time scales in Table 8 , where $F_{\text {var }}(0)$ is taken to be the minimum of the total $F_{\text {var }}$. The large best-fit $\chi^{2}$ in stars and galaxies implies that the best-fit variability time scales are quite uncertain. A longer duration of observation would be ideal to improve the structure function.

\subsection{Emission Lines}


The BPT diagram is constructed for the variable candidates to explore the narrow emission-line variability. Again the emission-line ratios $\log _{10}([\mathrm{~N} \mathrm{II}] / \mathrm{H} \alpha)$ vs. $\log _{10}([\mathrm{O} \mathrm{III}] / \mathrm{H} \beta)$ are considered.

In measuring the EW of an emission line, we fit to a given continuum-subtracted spectrum a single Gaussian function. The continuum is estimated by non-negative least square fitting (Lawson \& Hanson 1974), provided by Dobos et al. (2006) who used the Bruzual \& Charlot (2003) stellar population $1 \AA$-resolution models. In this way, the stellar absorption in the Balmer lines is implicitly taken into account in the best-fit stellar continuum. In measuring the uncertainty of an EW, the following steps are performed. Firstly, the uncertainty in the luminosity densities of the stellar continuum of the spectrum is calculated by using $\delta f_{\lambda}^{c}=\delta f_{\lambda}$, where $\delta$ denotes the uncertainty of the continuum flux density $f_{\lambda}^{c}$ and of the observed spectrum $f_{\lambda}$. The uncertainty in each EW is calculated to be $\sum_{\lambda_{R}} \delta\left(f_{\lambda}-f_{\lambda}^{c}\right) / f_{\lambda}^{c} d \lambda$, where $\lambda_{R}$ is the region of influence of the emission lines, taken to be around the line center $\pm 280 \mathrm{~km} \mathrm{~s}^{-1}$. Finally, the uncertainty in each of the line ratios $\log _{10}([\mathrm{~N} \mathrm{II}] / \mathrm{H} \alpha)$ and $\log _{10}([\mathrm{O} \mathrm{III}] / \mathrm{H} \beta)$ is propagated by using common error propagation formula.

The line ratios between the dim and bright phases of sources are found to be located very closely on the BPT diagram, as shown in Fig. 9. The emission-line ratios and their uncertainties for various object types are given in Table 9. The uncertainty in the flux measurement is small, for example in the star-forming galaxies, which indicates a reliable line measurements. For both line ratios the difference between the dim and bright phases is typically comparable to the $1 \sigma$ sample scatter in the line-ratio difference (i.e., the last two columns of Table. 9). This is true for all of the object types considered, meaning we find no evidence of narrow emissionline-ratio variability which is above $1 \sigma$ level. When dividing the line-ratio measurements by the first and second epoch (Fig. 10) instead of the dim and bright phases, we also see no difference between the two that is larger than $1 \sigma$. Further, the line ratios of the variable candidates and the non-variable candidates (Fig. 11) do not show substantial difference in the BPT diagram, true for all of the emission-line galaxy types.

The above analyses are also repeated with the intrinsically de-reddened spectra using the best-fit color excess $(\mathrm{E}(\mathrm{B}-\mathrm{V}))$ (Yip et al. 2008 in prep.), by the non-negative least square fitting of stellar population models on the SDSS DR6 galaxies (Dobos et al. 2006). Expectedly, each of the average line ratios remains unchanged to the $1 \sigma$ level (not shown), because the central wavelengths of the two lines for a given line ratio are close and is thus insensitive to dust reddening.

\section{COMPARISON WITH STAR AND QSO}

To evaluate further the performance of the calibration refinement, we refine the calibration in stellar and QSO spectra in a spectroscopic plate using calibration spectrum constructed from galaxies of the same plate. The procedure is described in $\S 4$.

\subsection{Variability in SDSS Stellar and QSO spectra}

Table 10 lists the statistics of $F_{\text {var }}$ in our sample of the SDSS stellar spectra. The improvement in the re- peatability after the SYS+BAND refinement is a factor of three. The average $F_{\text {var }}$ of stars is found to be slightly higher than that of the galaxies.

Table 11 lists the statistics of $F_{\text {var }}$ in our sample of the SDSS QSO spectra. The QSOs are located within a redshift range of $0.08-3.51$ with an average of 1.16 . Upon the $S F$ analysis the characteristic time scale of the QSOs is found to be $0.4 \pm 1.0$ years (Table 7 ), which agrees with the time scales commonly found in the literature (e.g., Cristiani et al. 1996; Ivezić et al. 2004; Vanden Berk et al. 2004) for the UV-optical variability.

The comparison between the $S F$ of galaxies, stars and QSOs in Fig. 6,7 shows that $F_{\text {var }}(\mathrm{QSO})>F_{\text {var }}($ star $)>$ $F_{\text {var }}$ (galaxy).

\subsection{Cross match with GCVS}

The full samples of galaxies, stars and QSOs are matched with the General Catalogue of Variable Stars (GCVS, Kholopov et al. 1999, Vol. I-III and references therein) as an assessment to the definition of variability. The RA and DEC are both matched to within $1^{\prime \prime}$, respectively.

Table 12 shows the objects that exist in both the GCVS and our samples. As expected, all of the matched objects are spectroscopically classified as stars by the SDSS. Three of the five GCVS stars are defined in this work to be variable candidates ( $>3 \sigma$ detection), hence a completeness of $60 \%$ when using 2 -epoch observations to infer the stellar variability.

The above indicates that the variability of stars and galaxies in this study could in principle be limited by the small number of epochs, also pointed out by Ivezić et al. (2003); and that the defining of variable stars by more than $3 \sigma$ over the average in the variability may need to be adjusted. The fact that more than half of the variable candidates are real variable stars according to the GCVS shows that the calibration refinement is successful in selecting those objects.

\subsection{Cross match with SDSS Southern Stripe}

Our full samples of galaxies, stars and QSOs are also cross-matched with the variable catalog constructed by Sesar et al. (2007) based on the SDSS repeated imaging (the Stripe 82). The data cover more observation epochs (from 4 to 28, with an average of 9), useful for determining the completeness of our variable candidates based on 2 epochs. The matched objects are found to be either stars or QSOs in the SDSS spectral classification, as Sesar et al. (2007)'s catalog is for un-resolved objects only. In Table 13 the matched stars are listed. Five out of 22 sources matched to the SDSS variable catalog are defined to be variable stars in this work ( $>3 \sigma$ detection), implying a $23 \%$ completeness when using 2-epoch observations to infer the stellar variability.

For QSOs, because more than 300 matches are found, only the number of found objects is summarized in Table 14, along with our detection significance as variable candidates. About $10 \%$ of the objects are considered to be real variables in this work. If some of the narrow-line AGNs do vary in time, the completeness of the variable candidates from our current 2-epoch sample may be as low as $10 \%$.

\section{SUMMARY}


We probe the spectroscopic variability in the galaxies and narrow-line AGNs in the optical wavelengths using multi-epoch observations in the SDSS. In order to detect the expected low amplitude of variability, we compare several approaches to refine the spectrophotometric calibration in the SDSS. The final calibration, in terms of the mean and the standard deviation of the variability in our galaxy sample, is a factor of two better than the official SDSS reductions.

Our sample of galaxy pairs spans in the restframe $\sim 110$ days on average, with a maximum of $\sim 700$ days. The average change in the narrow-line AGN continuum flux, when converted to synthetic AB absolute magnitudes using the SDSS filters, is $0.07 \pm 0.26 \mathrm{mag}$ in the synthetic $g$ band, where the uncertainty is propagated from the uncertainty in the spectral flux measurement. The fact that no obvious continuum variability is found is consistent with the expectation from the AGN unified model (Antonucci 1993; Urry \& Padovani 1995), in which some of the light-emitting (in our context, continuumgenerating) structures in a narrow-line AGN, such as the accretion disk, are obscured by a dusty torus. Hence, any variability in such structures (cf. the UV-optical variability in QSOs in the time scale of 1 year) is not observed. This also provides an empirical evidence for modeling the continua of narrow-line AGNs using stellar population models (e.g., Kauffmann et al. 2003; Hao et al. 2005). Further, if we use this continuum variability measurement to set an upper limit on the AGN activities, then the ratio between the flux of any varying spectral component, presumably related to AGN activities, and that of the host galaxy is at most $\sim 30 \%$.

By comparing the narrow emission-line ratios $\log _{10}\left(\left[\begin{array}{ll}\mathrm{N} & \mathrm{II}\end{array}\right] / \mathrm{H} \alpha\right)$ and $\log _{10}\left(\left[\begin{array}{ll}\mathrm{O} & \mathrm{III}\end{array}\right] / \mathrm{H} \beta\right)$ between the defined dim and bright phases in the continuum variable candidates, we find no evidence for their variability to be substantially larger than the $1 \sigma$ sample scatter in the line-ratio difference. This is consistent with the common view in which the narrow-line emissions in the AGNs are generated from low-density clouds. For example, an electron density $n_{e} \sim 1,000 \mathrm{~cm}^{-3}$ gives rise to a hydrogen recombination time $\sim 100$ years, which is typically much longer than the duration of the observation.

We found no evidence of continuum variability in galaxies of eClass types A, B, C, D that is larger than the variability uncertainty. This agrees with the expectation that stellar light dominates their continuum, as in the narrow-line AGNs.

We tabulate the upper limits of both the continuum and emission-line variability as a function of spectral type, using the classification schemes eClass and the BPT diagram. These values can serve as a sanity check for researchers who study variability for other sources.

\subsection{Variability in Classification}

There were few attempts in examining the galaxy classification from the point of view of variability. A recent work by Brunzendorf \& Meusinger (2001) suggested variability is an efficient method to find narrow-line AGN. However, this promising result was not subsequently confirmed by the authors (Meusinger \& Brunzendorf 2002). Our result on narrow emission-line ratios being nonvariable seems to agree with the latter claim made by the authors.

\subsection{Next Steps}

So far we have focused on the average variability properties of the galaxies and narrow-line AGNs. We are investigating, on the object-to-object basis, any dramatic variation in spectral features or types.

\section{ACKNOWLEDGEMENTS}

We thank Brian Wilhite, David Turnshek and Julian Krolik for discussions. We thank Brigitte König, Amy Kimball and Scot Kleinman for discussions on variable stars. We thank Ani Thakar for discussions on SDSS databases. We thank the referee for helpful suggestions, and discussions on narrow-line Seyfert 1 galaxies. ASS, RFGW, TB and CWY acknowledge support through grants from the W.M. Keck Foundation and the Gordon and Betty Moore Foundation, to establish a program of data-intensive science at the Johns Hopkins University. AJC acknowledges partial support from the NSF ITR award 0851007. LD and IC acknowledge support from grants MSRC-2005-038, NAP-2005/ KCKHA005 and MRTN-CT-2004-503929.

Funding for the SDSS and SDSS-II has been provided by the Alfred P. Sloan Foundation, the Participating Institutions, the National Science Foundation, the U.S. Department of Energy, the National Aeronautics and Space Administration, the Japanese Monbukagakusho, the Max Planck Society, and the Higher Education Funding Council for England. The SDSS Web Site is http://www.sdss.org/.

The SDSS is managed by the Astrophysical Research Consortium for the Participating Institutions. The Participating Institutions are the American Museum of Natural History, Astrophysical Institute Potsdam, University of Basel, University of Cambridge, Case Western Reserve University, University of Chicago, Drexel University, Fermilab, the Institute for Advanced Study, the Japan Participation Group, Johns Hopkins University, the Joint Institute for Nuclear Astrophysics, the Kavli Institute for Particle Astrophysics and Cosmology, the Korean Scientist Group, the Chinese Academy of Sciences (LAMOST), Los Alamos National Laboratory, the Max-Planck-Institute for Astronomy (MPIA), the MaxPlanck-Institute for Astrophysics (MPA), New Mexico State University, Ohio State University, University of Pittsburgh, University of Portsmouth, Princeton University, the United States Naval Observatory, and the University of Washington. 


\section{REFERENCES}

Abazajian, K., et al. 2003, AJ, 126, 2081

Adelman-McCarthy, J. K., et al. 2008, ApJS, 175, 297

Antonucci, R. 1993, ARA\&A, 31, 473

Aretxaga, I., Cid Fernandes, R., \& Terlevich, R. J. 1997, MNRAS, 286, 271

Baldwin, J. A., Phillips, M. M., \& Terlevich, R. 1981, PASP, 93, 5

Blanton, M. R., Lin, H., Lupton, R. H., Maley, F. M., Young, N., Zehavi, I., \& Loveday, J. 2003, AJ, 125, 2276

Bonoli, F., Braccesi, A., Federici, L., Zitelli, V., \& Formiggini, L. 1979, A\&AS, 35, 391

Bono, G., Trevese, D., \& Turatto, M. 2003, Memorie della Societa Astronomica Italiana, 74, 1004

Brunzendorf, J., \& Meusinger, H. 2001, A\&A, 373, 38

Bruzual, G., \& Charlot, S. 2003, MNRAS, 344, 1000

Connolly, A. J., Szalay, A. S., Bershady, M. A., Kinney, A. L., \& Calzetti, D. 1995, AJ, 110, 1071

Cristiani, S., Trentini, S., La Franca, F., Aretxaga, I., Andreani, P., Vio, R., \& Gemmo, A. 1996, A\&A, 306, 395

de Vries, W. H., Becker, R. H., \& White, R. L. 2003, AJ, 126, 1217

Dobos, L., Budavari, T., Csabai, I., \& Szalay, A. 2006, The Virtual Observatory in Action: New Science, New Technology, and Next Generation Facilities, 26th meeting of the IAU, Special Session 3, 17-18, 21-22 August, 2006 in Prague, Czech Republic, SPS3, \#76, 3

Fukugita, M., Ichikawa, T., Gunn, J. E., Doi, M., Shimasaku, K., \& Schneider, D. P. 1996, AJ, 111, 1748

Giannuzzo, E. M., \& Stirpe, G. M. 1996, A\&A, 314, 419

Goodrich, R. W. 1989, ApJ, 340, 190

Guainazzi, M., Matt, G., Antonelli, L. A., Fiore, F., Piro, L., \& Ueno, S. 1998, MNRAS, 298, 824

Gunn, J. E., et al. 1998, AJ, 116, 3040

Gunn, J. E., et al. 2006, AJ, 131, 2332

Hao, L., et al. 2005, AJ, 129, 1783

Hawkins, M. R. S. 1993, Nature, 366, 242

Heckman, T. M. 1980, A\&A, 87, 152

Hogg, D. W., Finkbeiner, D. P., Schlegel, D. J., \& Gunn, J. E. 2001, AJ, 122, 2129

Hopkins, A. M., et al. 2003, ApJ, 599, 971

Hughes, P. A., Aller, H. D., \& Aller, M. F. 1992, ApJ, 396, 469

Ivezić, Ž., et al. 2003, Memorie della Societa Astronomica Italiana, 74, 978

Ivezić, Ž., et al. 2004, Astronomische Nachrichten, 325, 583

Ivezić, Ž., et al. 2004, The Interplay Among Black Holes, Stars and ISM in Galactic Nuclei, 222, 525

Jang, M. 2001, Ap\&SS, 275, 209

Kauffmann, G., et al. 2003, MNRAS, 346, 1055

Kawaguchi, T., Mineshige, S., Umemura, M., \& Turner, E. L. 1998, ApJ, 504, 671

Kennicutt, R. C., Jr. 1992, ApJS, 79, 255

Kewley, L. J., Groves, B., Kauffmann, G., \& Heckman, T. 2006, MNRAS, 372, 961

Kholopov, P. N., et al. 1999, VizieR Online Data Catalog, 2214, 0

Komossa, S. 2008, Revista Mexicana de Astronomia y Astrofisica Conference Series, 32, 86

Lawson, C. L. and Hanson, R. J., Solving Least Square Problem 1974, Prentice-Hall

Lupton, R., Gunn, J. E., Ivezić, Z., Knapp, G. R., \& Kent, S. 2001, Astronomical Data Analysis Software and Systems X, 238,269

Madgwick, D. S., Somerville, R., Lahav, O., \& Ellis, R. 2003, MNRAS, 343, 871

Mateos, S., Barcons, X., Carrera, F. J., Page, M. J., Ceballos, M. T., Hasinger, G., \& Fabian, A. C. 2007, A\&A, 473, 105

Meusinger, H., \& Brunzendorf, J. 2002, A\&A, 390, 439

Miller, C. J., Nichol, R. C., Gómez, P. L., Hopkins, A. M., \& Bernardi, M. 2003, ApJ, 597, 142
Mueller, M., Madejski, G. M., Done, C., \& Zycki, P. T. 2003 Bulletin of the American Astronomical Society, 35, 637 O'Donnell, J. E. 1994, ApJ, 422, 158

Oke, J. B., \& Gunn, J. E. 1983, ApJ, 266, 713

Osterbrock, D. E. 1989, Astrophysics of gaseous nebulae and active galactic nuclei, University Science Books, 1989

Osterbrock, D. E., \& Pogge, R. W. 1985, ApJ, 297, 166

Pereyra, N. A., Vanden Berk, D. E., Turnshek, D. A., Hillier, D. J., Wilhite, B. C., Kron, R. G., Schneider, D. P., \& Brinkmann, J. 2006, ApJ, 642, 87

Peterson, B. M., Pogge, R. W., Wanders, I., Smith, S. M., \& Romanishin, W. 1995, PASP, 107, 579

Peterson, B. M., Wanders, I., Bertram, R., Hunley, J. F., Pogge, R. W., \& Wagner, R. M. 1998, ApJ, 501, 82

Peterson, B. M., McHardy, I. M., \& Wilkes, B. J. 2000, New Astronomy Review, 44, 491

Peterson, B. M. 2001, Advanced Lectures on the Starburst-AGN, 3

Pier, J. R., Munn, J. A., Hindsley, R. B., Hennessy, G. S., Kent, S. M., Lupton, R. H., \& Ivezić, Ž. 2003, AJ, 125, 1559

Richards, G. T., et al. 2002, AJ, 123, 2945

Rodriguez-Pascual, P. M., et al. 1997, ApJS, 110, 9

Schlegel, D. J., Finkbeiner, D. P., \& Davis, M. 1998, ApJ, 500, 525

Sesar, B., et al. 2006, AJ, 131, 2801

Sesar, B., et al. 2007, AJ, 134, 2236

Simonetti, J. H., Cordes, J. M., \& Heeschen, D. S. 1985, ApJ, 296, 46

Smith, J. A., et al. 2002, AJ, 123, 2121

Spitzer, L. 1978, New York Wiley-Interscience, 1978. 333

Stalin, C. S., Gopal-Krishna, Sagar, R., \& Wiita, P. J. 2004, MNRAS, 350, 175

Stoughton, C., et al. 2002, AJ, 123, 485

Strauss, M. A., et al. 2002, AJ, 124, 1810

SubbaRao, M., Frieman, J., Bernardi, M., Loveday, J., Nichol, B., Castander, F., \& Meiksin, A. 2002, Proc. SPIE, 4847, 452

Tohline, J. E., \& Osterbrock, D. E. 1976, ApJ, 210, L117

Trevese, D., Kron, R. G., Majewski, S. R., Bershady, M. A., \& Koo, D. C. 1994, ApJ, 433, 494

Trippe, M. L., Crenshaw, D. M., Deo, R., \& Dietrich, M. 2008 , AJ, 135, 2048

Tucker, D. L., et al. 2006, Astronomische Nachrichten, 327, 821

Urry, C. M., \& Padovani, P. 1995, PASP, 107, 803

Vanden Berk, D. E., et al. 2001, AJ, 122, 549

Vanden Berk, D. E., et al. 2004, ApJ, 601, 692

Vaughan, S., Edelson, R., Warwick, R. S., \& Uttley, P. 2003, MNRAS, 345, 1271

Veilleux, S., \& Osterbrock, D. E. 1987, ApJS, 63, 295

Veilleux, S., Kim, D.-C., Sanders, D. B., Mazzarella, J. M., \& Soifer, B. T. 1995, ApJS, 98, 171

Wanders, I., Peterson, B. M., Pogge, R. W., Derobertis, M. M., \& van Groningen, E. 1992, A\&A, 266, 72

Wang, J., \& Wei, J. Y. 2008, ApJ, 679, 86

Wilhite, B. C., Vanden Berk, D. E., Kron, R. G., Schneider, D. P., Pereyra, N., Brunner, R. J., Richards, G. T., \& Brinkmann, J. V. 2005, ApJ, 633, 638

Wilhite, B. C., Vanden Berk, D. E., Brunner, R. J., \&

Brinkmann, J. V. 2006, ApJ, 641, 78

Winkler, H., Glass, I. S., van Wyk, F., Marang, F., Jones, J. H. S., Buckley, D. A. H., \& Sekiguchi, K. 1992, MNRAS, 257, 659

Yip, C. W., et al. 2004, AJ, 128, 585

York, D. G., et al. 2000, AJ, 120, 1579

Zhou, H., Wang, T., Yuan, W., Lu, H., Dong, X., Wang, J., \& Lu, Y. 2006, ApJS, 166, 128 


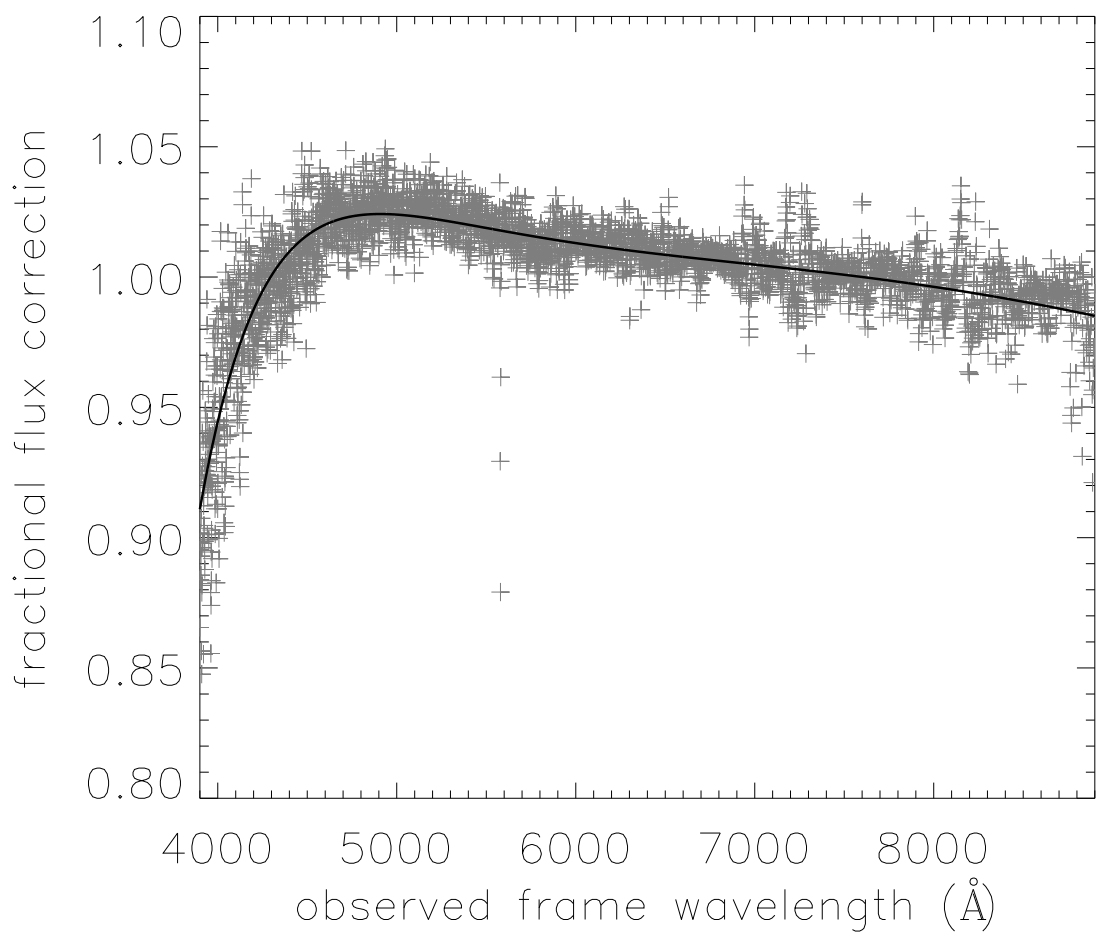

FIG. 1. - The calibration spectrum (gray crosses) of the spectroscopic plate 390 and its 5th-order polynomial fit (black line). The correction in flux density between two epochs is a function of the observed-frame wavelength. 

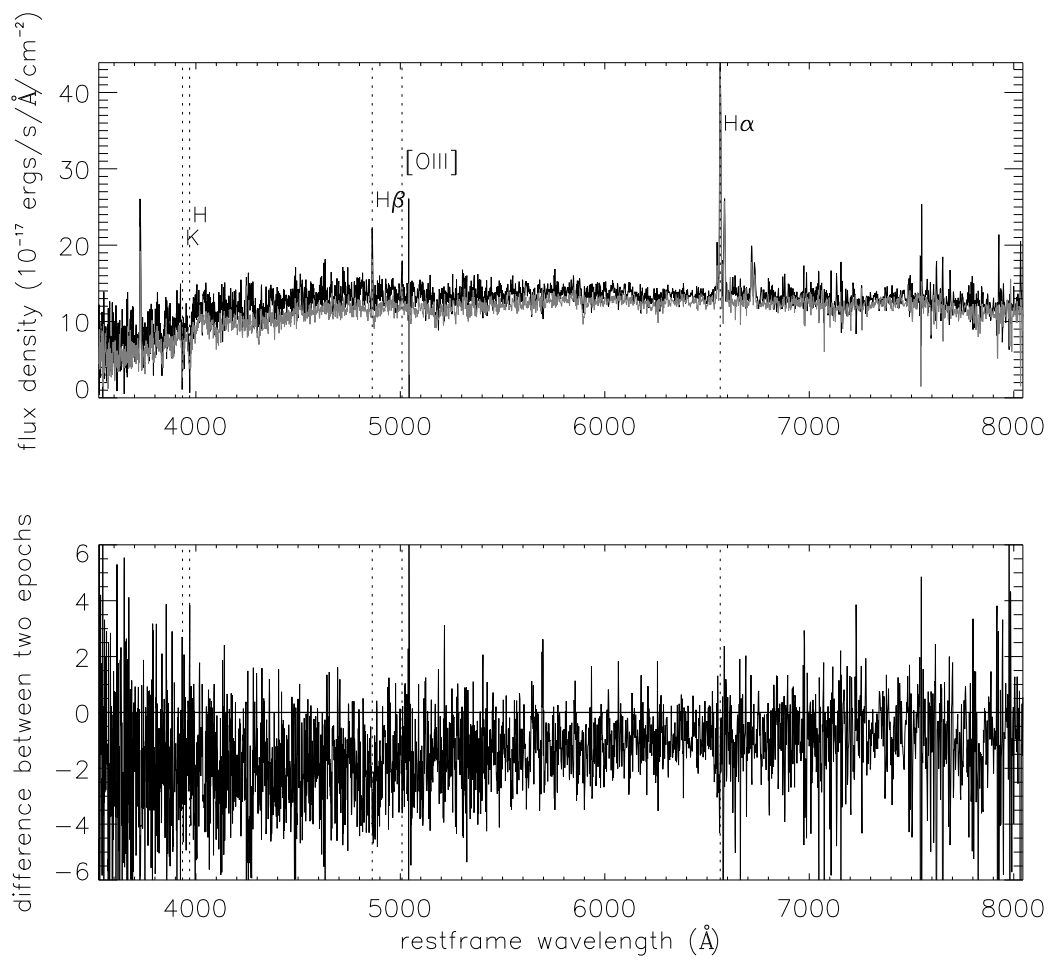

FIG. 2.- An example star-forming galaxy without any calibration refinement $\left(F_{\text {var }}=0.058\right)$. Top: spectra at the first (black) and the second (gray) epoch. Bottom: difference spectrum.
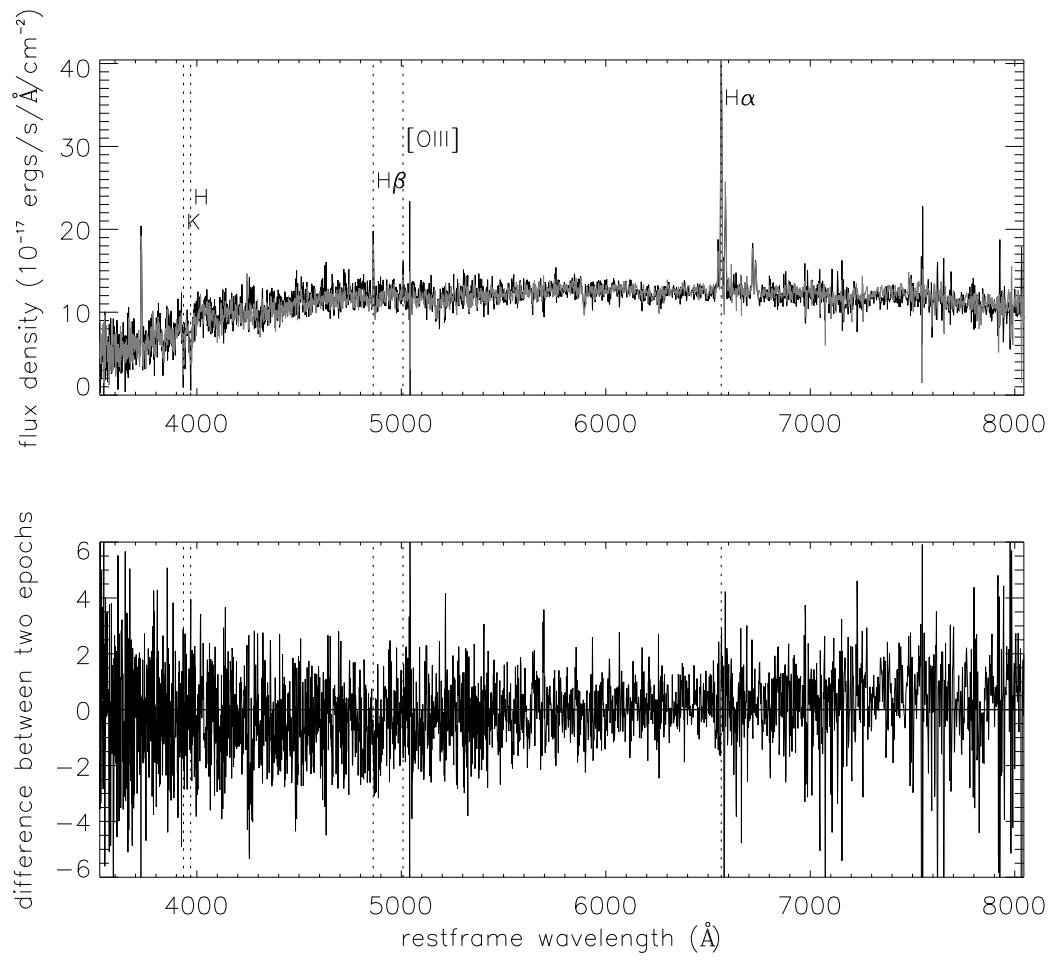

FIG. 3.- The same galaxy as in Fig. 2, which shows zero continuum variability $\left(F_{\mathrm{var}}=0\right)$ after the SYS+BAND calibration refinement. Top: spectra at the first (black) and the second (gray) epoch. Bottom: difference spectrum. 

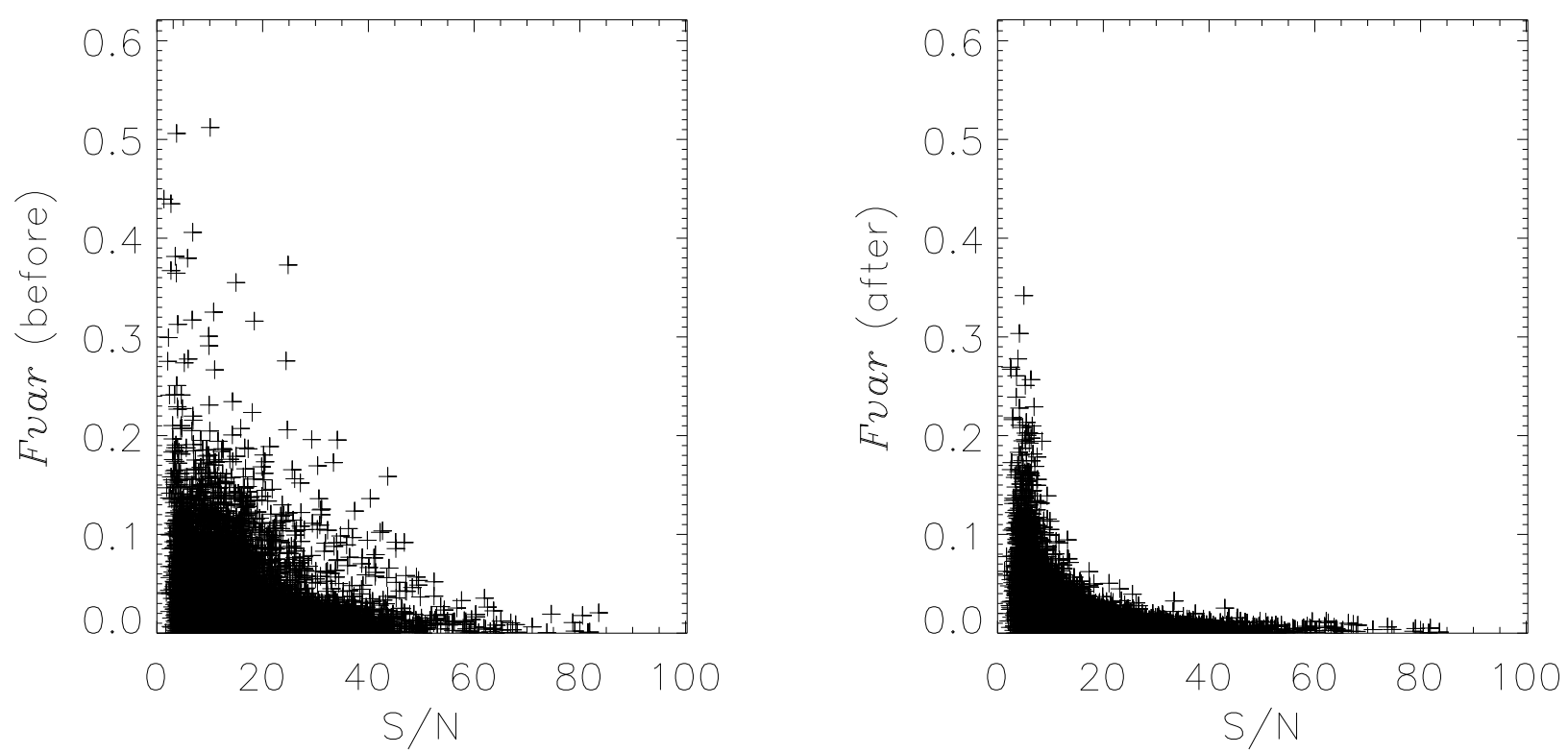

FIG. 4. - $F_{\text {var }}$ vs. S/N of galaxy spectra before (left) and after (right) the SYS+BAND calibration refinement. The number of objects in each spectroscopic plate is listed in Table 1.

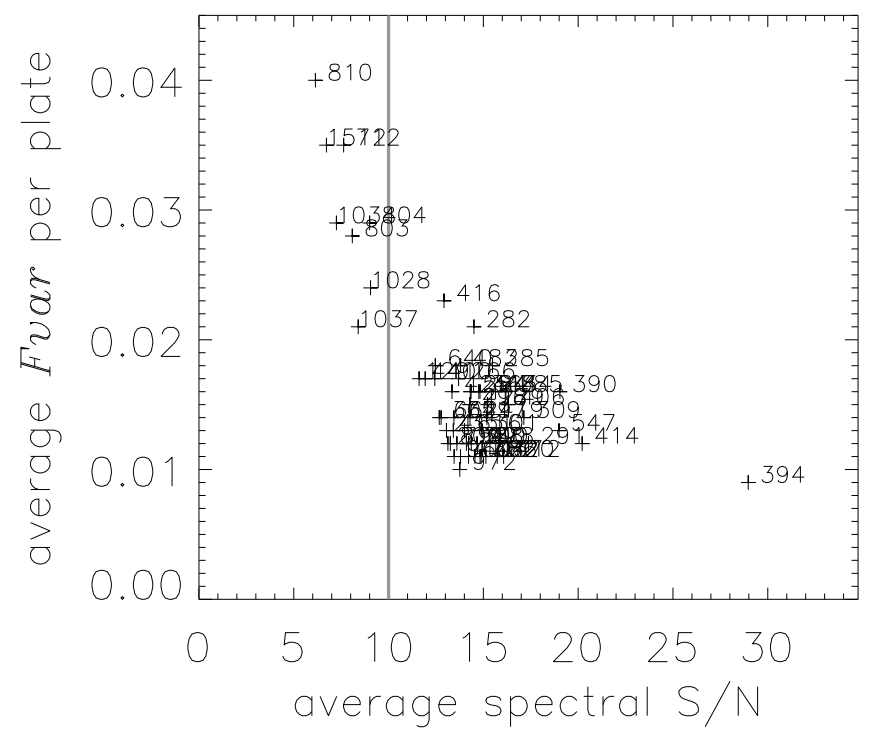

FIG. 5.- The average variability of galaxy spectra over a given spectroscopic plate plotted versus the average spectral S/N. The spectra at $\mathrm{S} / \mathrm{N} \geq 10$ are selected in the subsequent definition of variable and non-variable candidates to minimize variation in $F_{\text {var }}$ among plates. 


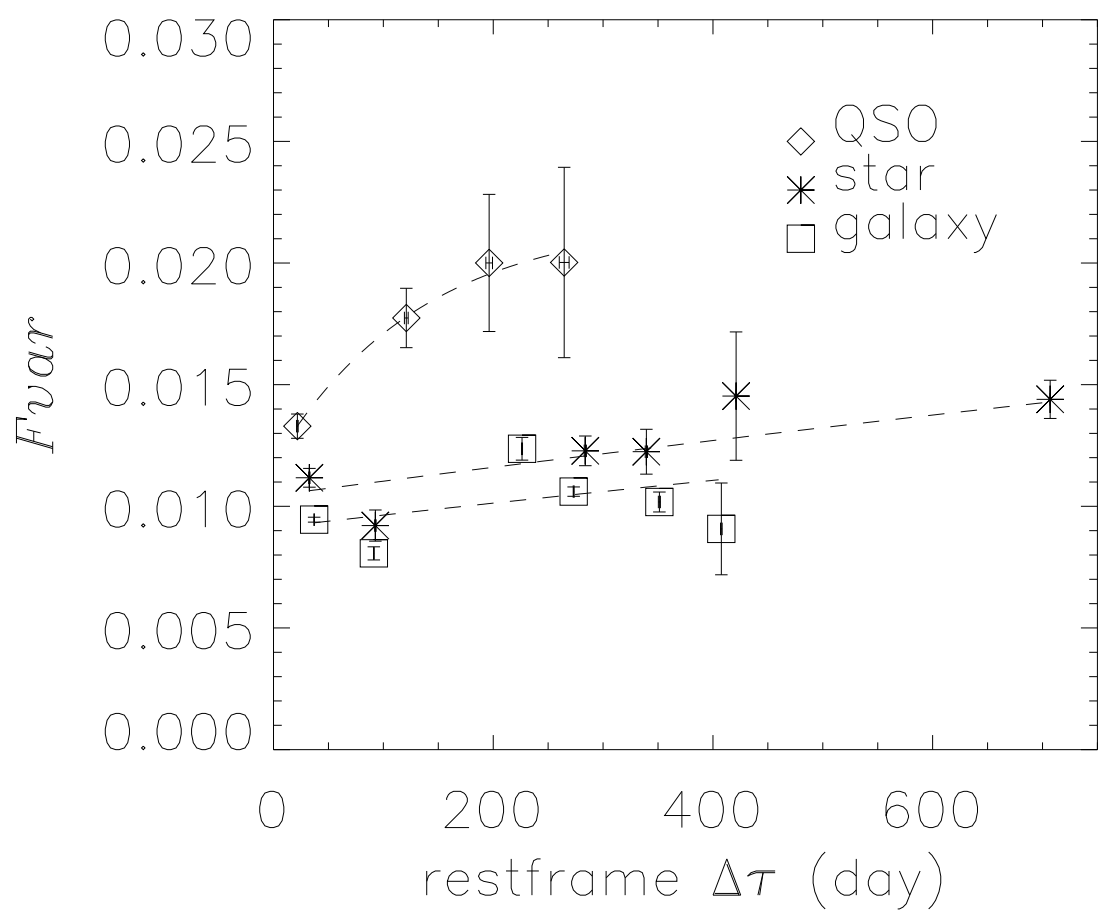

FIG. 6.- Restframe structure function of the QSOs, stars and galaxies. Only spectra with $\mathrm{S} / \mathrm{N} \geq 10$ are shown. Error bar is $1 \sigma_{m}$ for each axis. Dotted line shows the best-fit structure function of an exponential functional form in $\Delta \tau_{\text {rest }}$.

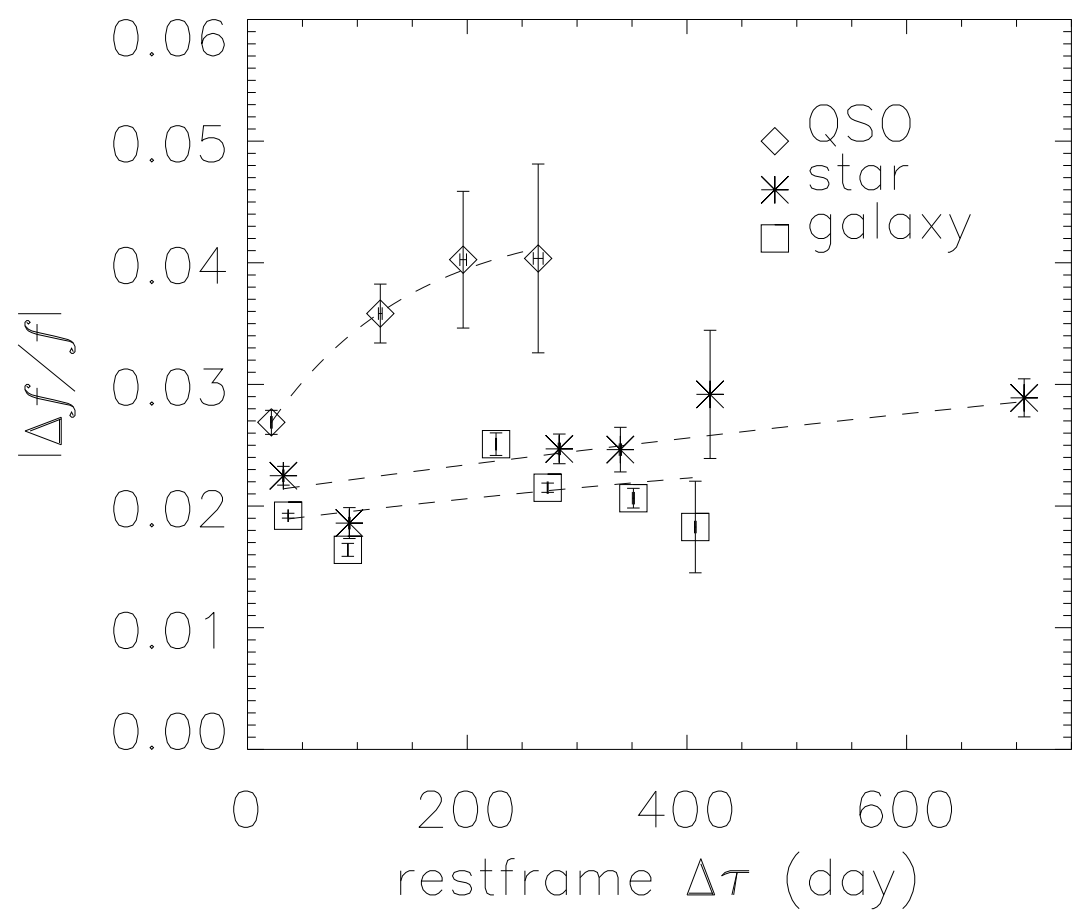

FIG. 7.- Restframe structure function. Same as Fig. 6 except that the fraction change in total flux $|\Delta f / f|$ is used instead of $F_{\text {var }}$. 


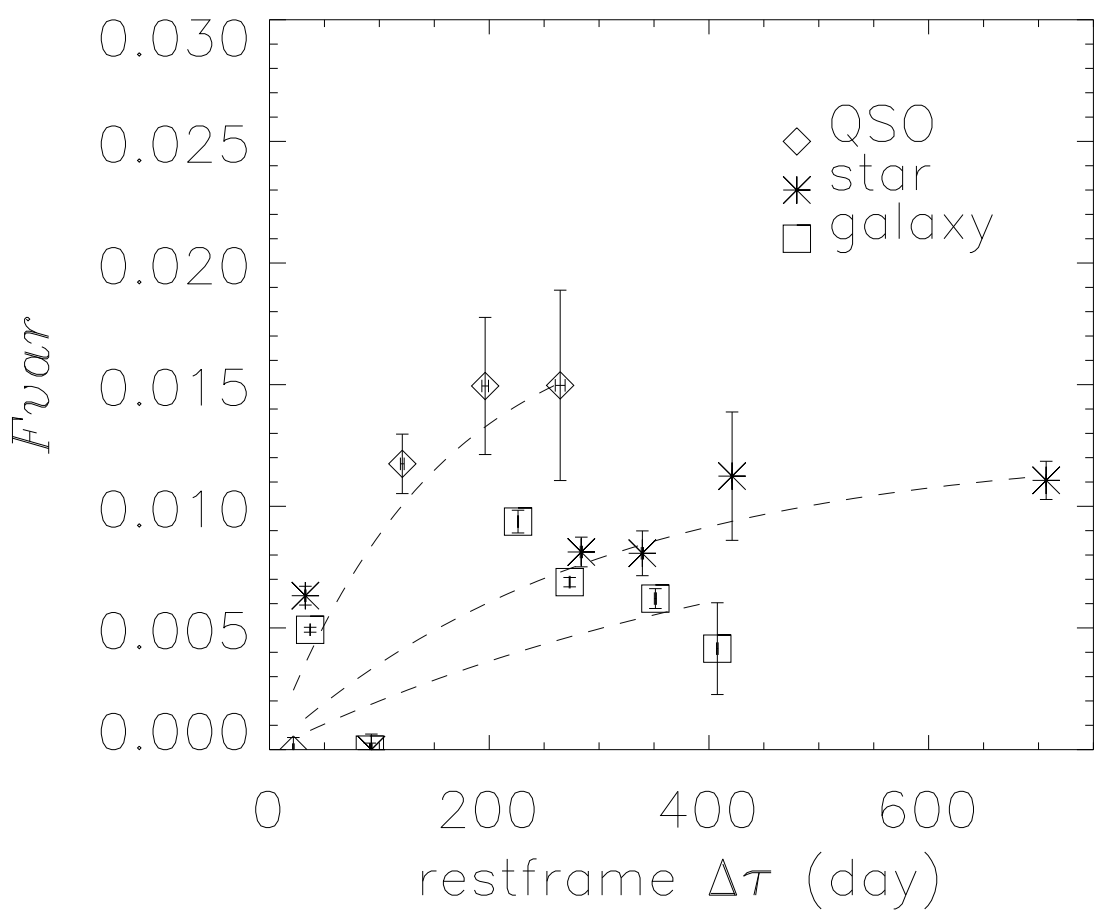

FIG. 8.- Restframe structure function of the QSOs, stars and galaxies. Different from Fig. 6 which shows the total variability, here the variability at the zero time lag is quadrature subtracted, respectively in each spectral type. Only spectra with $\mathrm{S} / \mathrm{N} \geq 10$ are shown. Error bar is $1 \sigma_{m}$ for each axis. Dotted line shows the best-fit structure function of an exponential functional form in $\Delta \tau_{\text {rest }}$. 


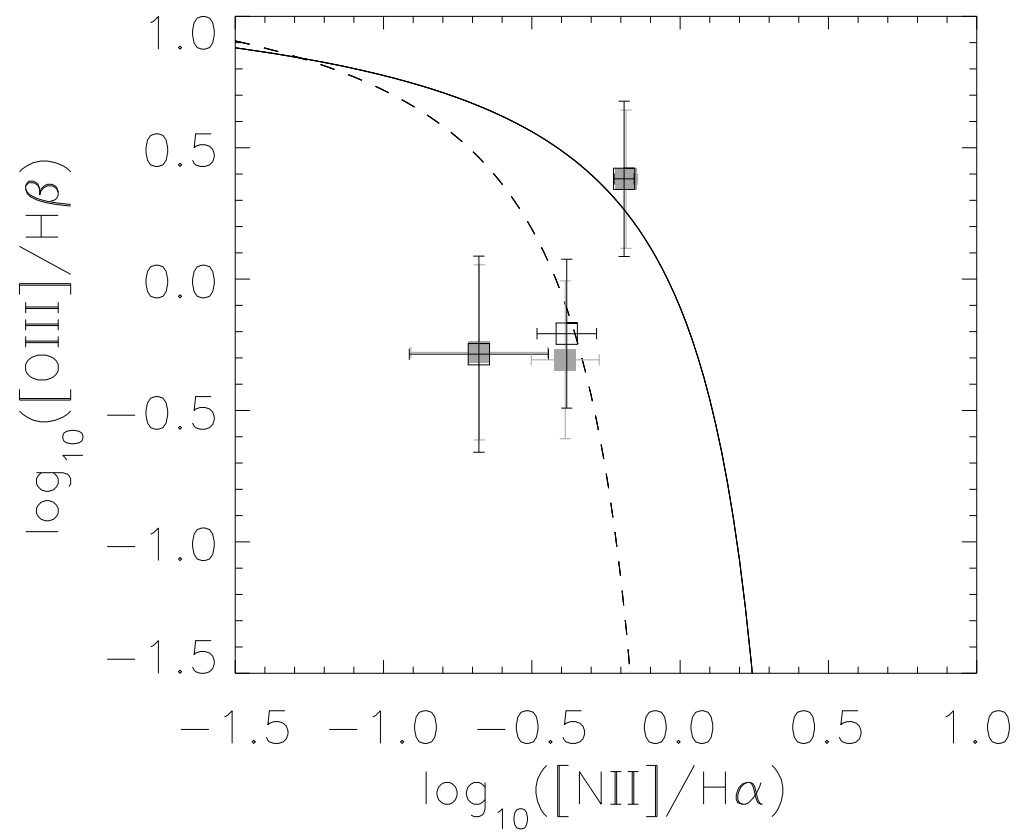

FIG. 9. - The BPT diagram of the continuum variable candidates. The object type from the lowest to the highest [N II]/H $\alpha$ value is: star-forming, composite and Seyfert 2. In each duo the filled square represents the average line ratios of the brighter objects, and the empty square for the dimmer ones. The solid and dashed lines respectively define the Seyfert 2 and star-forming galaxies as given by Kewley et al. (2006). The line ratios in all object types do not show substantial change between both epochs. The error bar represents the $1 \sigma$ sample scatter in each phase.

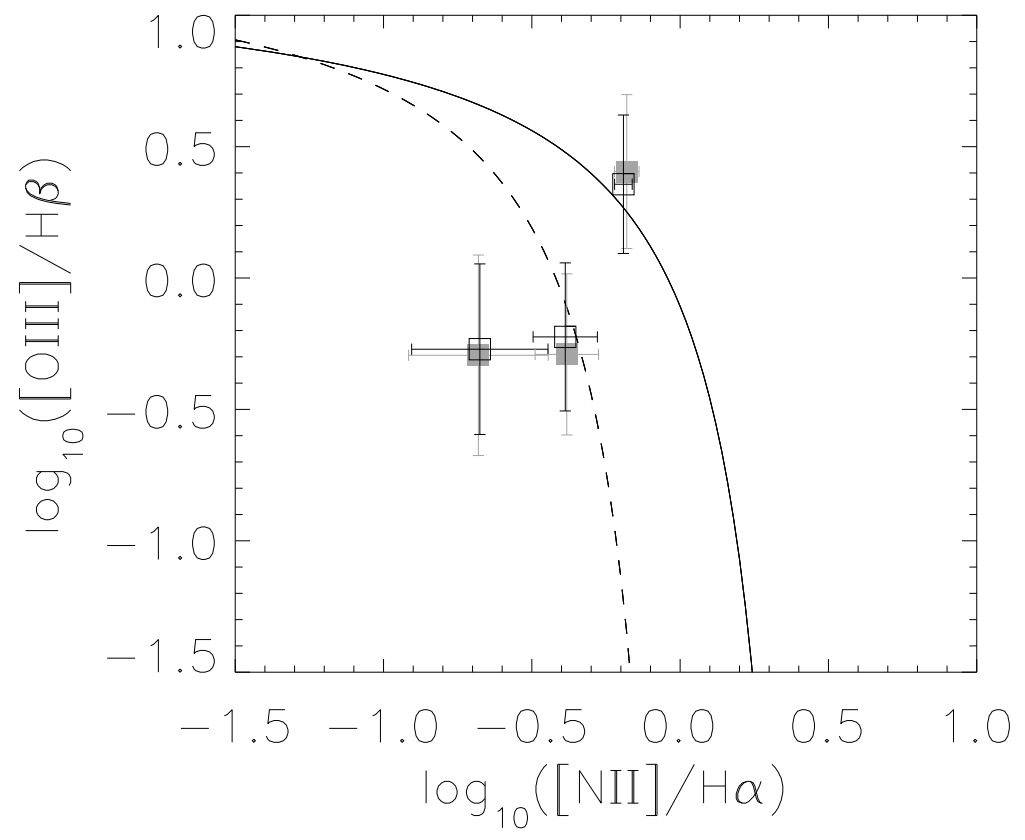

FIG. 10. - Similar to Fig. 9, but in each duo the filled square represents the average line ratios of the first epoch, and the empty square for the latter epoch. The error bar represents the $1 \sigma$ sample scatter in each epoch. 


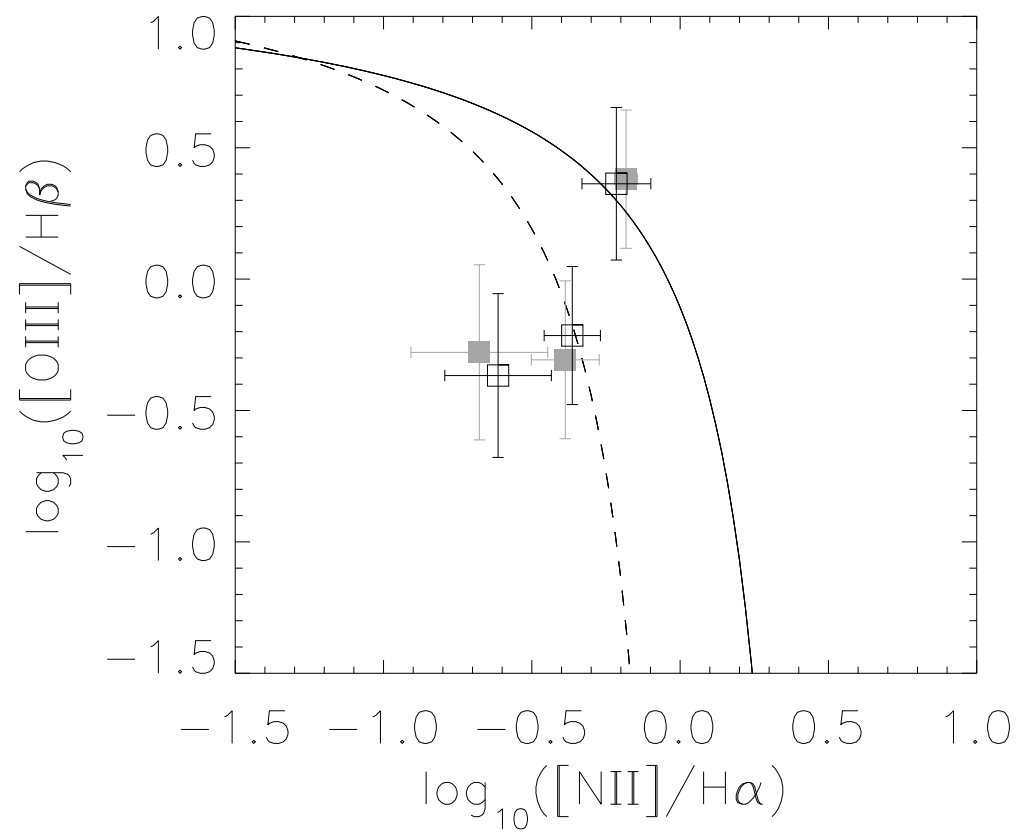

Fig. 11. - Similar to Fig. 9, but in each duo the filled square represents the average line ratios of the continuum variable candidates, and the empty square for the non-variable ones. Line ratios in both cases are from the bright phase. The error bar represents the $1 \sigma$ sample scatter. 
TABLE 1 Spectroscopic plates used in the analysis of galaxies.

\begin{tabular}{|c|c|c|c|c|c|}
\hline plate $^{\mathrm{a}, \mathrm{b}}$ & first epoch MJD & second epoch MJD & difference in MJD & 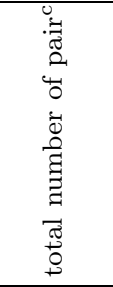 & 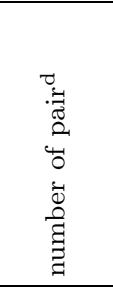 \\
\hline 266 & 51602 & 51630 & 28 & 447 & 356 \\
\hline 279 & 51608 & 51984 & 376 & 430 & 361 \\
\hline 282 & 51630 & 51658 & 28 & 346 & 278 \\
\hline 284 & 51662 & 51943 & 281 & 425 & 336 \\
\hline 285 & 51663 & 51930 & 267 & 417 & 317 \\
\hline 291 & 51660 & 51928 & 268 & 444 & 344 \\
\hline 296 & 51665 & 51984 & 319 & 448 & 364 \\
\hline 297 & 51663 & 51959 & 296 & 453 & 369 \\
\hline 300 & 51666 & 51943 & 277 & 386 & 288 \\
\hline 301 & 51641 & 51942 & 301 & 370 & 282 \\
\hline 304 & 51609 & 51957 & 348 & 398 & 317 \\
\hline 306 & 51637 & 51690 & 53 & 435 & 352 \\
\hline 309 & 51666 & 51994 & 328 & 371 & 287 \\
\hline 340 & 51691 & 51990 & 299 & 385 & 302 \\
\hline 348 & 51671 & 51696 & 25 & 313 & 240 \\
\hline 351 & 51695 & 51780 & 85 & 395 & 311 \\
\hline 385 & 51783 & 51877 & 94 & 326 & 259 \\
\hline 390 & 51816 & 51900 & 84 & 392 & 313 \\
\hline 394 & 51812 & 51913 & 101 & 347 & 267 \\
\hline 404 & 51812 & 51877 & 65 & 421 & 335 \\
\hline 406 & 51817 & 52238 & 421 & 319 & 239 \\
\hline 410 & 51816 & 51877 & 61 & 309 & 243 \\
\hline 411 & 51817 & 51873 & 56 & 398 & 318 \\
\hline 412 & 51931 & 52258 & 327 & 396 & 306 \\
\hline 413 & 51821 & 51929 & 108 & 351 & 274 \\
\hline 414 & 51869 & 51901 & 32 & 383 & 299 \\
\hline 415 & 51810 & 51879 & 69 & 366 & 277 \\
\hline 416 & 51811 & 51885 & 74 & 332 & 259 \\
\hline 418 & 51817 & 51884 & 67 & 367 & 285 \\
\hline 419 & 51812 & 51879 & 67 & 355 & 293 \\
\hline 422 & 51811 & 51878 & 67 & 465 & 375 \\
\hline 425 & 51884 & 51898 & 14 & 370 & 290 \\
\hline 437 & 51869 & 51876 & 7 & 304 & 235 \\
\hline 440 & 51885 & 51912 & 27 & 425 & 331 \\
\hline 483 & 51902 & 51942 & 40 & 324 & 247 \\
\hline 547 & 51959 & 52207 & 248 & 347 & 288 \\
\hline 588 & 52029 & 52045 & 16 & 435 & 344 \\
\hline 594 & 52027 & 52045 & 18 & 355 & 285 \\
\hline 616 & 52374 & 52442 & 68 & 450 & 349 \\
\hline 640 & 52178 & 52200 & 22 & 405 & 323 \\
\hline 644 & 52149 & 52173 & 24 & 408 & 339 \\
\hline 662 & 52147 & 52178 & 31 & 455 & 358 \\
\hline 712 & 52179 & 52199 & 20 & 216 & 131 \\
\hline 790 & 52433 & 52441 & 8 & 402 & 323 \\
\hline 803 & 52264 & 52318 & 54 & 430 & 355 \\
\hline 804 & 52266 & 52286 & 20 & 422 & 354 \\
\hline 810 & 52326 & 52672 & 346 & 502 & 360 \\
\hline 814 & 52370 & 52443 & 73 & 363 & 279 \\
\hline 820 & 52433 & 52438 & 5 & 307 & 242 \\
\hline 960 & 52425 & 52466 & 41 & 427 & 354 \\
\hline 972 & 52428 & 52435 & 7 & 417 & 345 \\
\hline 978 & 52431 & 52441 & 10 & 328 & 262 \\
\hline 1028 & 52562 & 52884 & 322 & 466 & 371 \\
\hline 1034 & 52525 & 52813 & 288 & 471 & 385 \\
\hline 1037 & 52826 & 52878 & 52 & 508 & 417 \\
\hline 1291 & 52735 & 52738 & 3 & 421 & 337 \\
\hline 1512 & 53035 & 53742 & 707 & 144 & 99 \\
\hline 1782 & 53299 & 53383 & 84 & 457 & 373 \\
\hline 2009 & 53857 & 53904 & 47 & 449 & 354 \\
\hline 2351 & 53772 & 53786 & 14 & 332 & 259 \\
\hline$\cdots$ & $\cdots$ & $\cdots$ & $\cdots$ & $23,330^{\mathrm{e}}$ & $18,435^{\mathrm{e}}$ \\
\hline
\end{tabular}

1 There are 60 spectroscopic plates used, taken from the SDSS DR6

2 The plates 1664, 1905, 1907, 2075 (not listed in the table) are not used because the number of galaxy pairs is less than 10. 
3 The total number of galaxy pairs in a plate.

4 The number of galaxy pairs with successful calibration refinement using SYS+BAND.

5 The number of galaxy pairs summing over all plates, respectively. 
TABLE 2

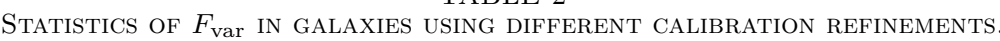

\begin{tabular}{|c|c|c|c|}
\hline method & mean $^{\mathrm{a}}$ & $\sigma_{m}$ & $\sigma$ \\
\hline No refinement ${ }^{b}$ & 0.020 & 0.0002 & 0.025 \\
\hline SYS+BAND & 0.010 & 0.0001 & 0.009 \\
\hline SYS+BAND $\left(280 \mathrm{~km} \mathrm{~s}^{-1}\right)^{\mathrm{c}}$ & 0.012 & 0.0001 & 0.011 \\
\hline SYS+OIII & 0.016 & 0.0001 & 0.015 \\
\hline SYS+BAND (RED galaxies)d & 0.010 & 0.0001 & 0.009 \\
\hline SYS+BAND (BLUE galaxies) & 0.010 & 0.0001 & 0.009 \\
\hline
\end{tabular}

NoтE. - There are 11,766 common spectral pair in each of the calibration refinement approaches. Each spectrum is chosen to be refined successfully in each and every of the above procedures. In principal there can be more objects with successful refinement in each case.

aThe statistics of $F_{\text {var }}$ are calculated at spectral $\mathrm{S} / \mathrm{N} \geq 10: \sigma_{m}$ is the standard deviation of the mean of $F_{\text {var }}, \sigma$ is the one sigma sample scatter.

bThe spectra are the direct output made available by the SDSS and no calibration refinement is done.

'Same as SYS+BAND (in which the rest-band considered is $6450 \AA \pm 520 \mathrm{~km} \mathrm{~s}^{-1}$ ) but using $6450 \AA \pm 280 \mathrm{~km} \mathrm{~s}^{-1}$.

${ }^{\mathrm{d}}$ Same as SYS+BAND but using only the RED or the BLUE galaxies in calculating the calibration spectrum.

TABLE 3

UPPER PERCENTILE OF THE GALAXY VARIABle CANDIDATES ${ }^{\mathrm{a}}$.

\begin{tabular}{lccccc}
\hline lower S/N & 0 & 5 & 10 & 15 & 20 \\
upper S/N & 5 & 10 & 15 & 20 & 40 \\
\hline number $^{\mathrm{b}}$ & 11 & 102 & 246 & 206 & 86 \\
percentile $^{\mathrm{c}}$ & 1.53 & 1.89 & 3.92 & 6.26 & 3.44 \\
\hline
\end{tabular}

Note. - In the galaxy sample using SYS+BAND refinement.

${ }^{\text {a }}$ The variable candidates are selected by $F_{\mathrm{var}}>($ mean $+3 \sigma)$ of $F_{\mathrm{var}}$ at a given spectral S/N.

${ }^{\mathrm{b}}$ The number of variable candidates between the lower and upper $\mathrm{S} / \mathrm{N}$.

cThe percentile of the corresponding number within a given $\mathrm{S} / \mathrm{N}$ range.

TABLE 4

Statistics of $F_{\text {var IN THE FUll Galaxy SAMPle. }}$

\begin{tabular}{|c|c|c|c|c|}
\hline type & number ${ }^{\mathrm{a}, \mathrm{b}}$ & mean & $\sigma_{m}$ & $\sigma^{\mathrm{a}}$ \\
\hline Seyfert 2 & 92 & 0.009 & 0.0008 & 0.008 \\
\hline Starforming & 1333 & 0.012 & 0.0003 & 0.011 \\
\hline Composite & 314 & 0.010 & 0.0005 & 0.009 \\
\hline eClass A & 729 & 0.010 & 0.0004 & 0.010 \\
\hline eClass B & 919 & 0.010 & 0.0003 & 0.010 \\
\hline eClass C & 587 & 0.011 & 0.0004 & 0.010 \\
\hline eClass D & 483 & 0.012 & 0.0005 & 0.010 \\
\hline eClass E & 3 & 0.024 & 0.0100 & 0.017 \\
\hline eClass F & 5 & 0.044 & 0.0124 & 0.028 \\
\hline RED & 9603 & 0.009 & 0.0001 & 0.009 \\
\hline BLUE & 2705 & 0.012 & 0.0002 & 0.011 \\
\hline
\end{tabular}

Note. - In the galaxy sample using SYS+BAND refinement.

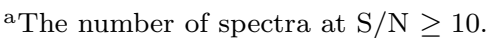

${ }^{b}$ We do not find any LINER (Heckman 1980) in our galaxy sample, using the classification criteria by Kewley et al. (2006, their Eqn. 15). 
TABLE 5

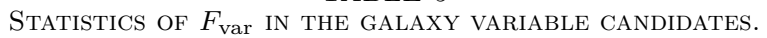

\begin{tabular}{lcccc}
\hline type & number $^{\mathrm{a}}$ & mean & $\sigma_{m}$ & $\sigma^{\mathrm{a}}$ \\
\hline Seyfert 2 & 5 & 0.026 & 0.0030 & 0.007 \\
Starforming & 62 & 0.037 & 0.0023 & 0.018 \\
Composite & 13 & 0.031 & 0.0043 & 0.015 \\
\hline eClass A & 34 & 0.031 & 0.0027 & 0.016 \\
eClass B & 45 & 0.031 & 0.0020 & 0.014 \\
eClass C & 37 & 0.030 & 0.0015 & 0.009 \\
eClass D & 18 & 0.034 & 0.0030 & 0.013 \\
\hline RED & 388 & 0.028 & 0.0006 & 0.012 \\
BLUE & 154 & 0.034 & 0.0012 & 0.015 \\
\hline
\end{tabular}

Note. - In the galaxy sample using SYS+BAND refinement.

aThe number of spectra at $\mathrm{S} / \mathrm{N} \geq 10$.

TABLE 6

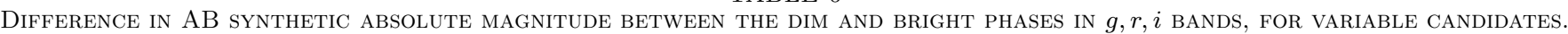

\begin{tabular}{|c|c|c|c|c|c|c|c|c|c|c|c|c|c|}
\hline type & $\left\langle M_{g}\right\rangle^{\mathrm{a}}$ & $\left\langle M_{r}\right\rangle$ & $\left\langle M_{i}\right\rangle$ & $\langle z\rangle^{\mathrm{b}}$ & $\left\langle\Delta M_{g}\right\rangle^{\mathrm{c}}$ & $\left\langle\Delta M_{r}\right\rangle$ & $\left\langle\Delta M_{i}\right\rangle$ & 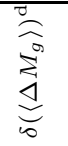 & 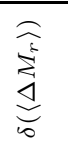 & 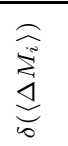 & 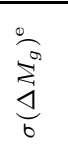 & $\underset{b}{\stackrel{E}{d}}$ & 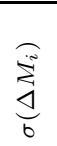 \\
\hline Seyfert 2 & -20.57 & -21.36 & -21.30 & 0.14 & 0.07 & 0.05 & 0.05 & 0.26 & 0.12 & 0.20 & 0.01 & 0.03 & 0.03 \\
\hline Starforming & -18.79 & -19.37 & -19.46 & 0.09 & 0.10 & 0.05 & 0.05 & 0.09 & 0.04 & 0.08 & 0.05 & 0.03 & 0.05 \\
\hline Composite & -19.79 & -20.53 & -20.53 & 0.11 & 0.09 & 0.04 & 0.03 & 0.19 & 0.08 & 0.13 & 0.05 & 0.02 & 0.03 \\
\hline eClass A & -20.70 & -21.55 & -21.25 & 0.15 & 0.08 & 0.05 & 0.06 & 0.11 & 0.05 & 0.85 & 0.04 & 0.04 & 0.05 \\
\hline eClass B & -20.12 & -20.95 & -20.74 & 0.13 & 0.09 & 0.05 & 0.04 & 0.10 & 0.04 & 4.82 & 0.03 & 0.03 & 0.03 \\
\hline eClass C & -19.95 & -20.73 & -20.81 & 0.12 & 0.09 & 0.04 & 0.04 & 0.12 & 0.05 & 0.08 & 0.03 & 0.02 & 0.03 \\
\hline eClass D & -18.18 & -18.75 & -18.91 & 0.07 & 0.09 & 0.04 & 0.06 & 0.16 & 0.07 & 0.12 & 0.05 & 0.02 & 0.06 \\
\hline RED & -20.14 & -20.99 & -20.91 & 0.13 & 0.08 & 0.04 & 0.05 & 0.03 & 0.01 & 3.16 & 0.04 & 0.03 & 0.04 \\
\hline BLUE & -19.27 & -19.87 & -19.89 & 0.10 & 0.09 & 0.05 & 0.05 & 0.06 & 0.03 & 0.05 & 0.05 & 0.03 & 0.05 \\
\hline
\end{tabular}

aThe sample-averaged absolute AB magnitude in the dim phase. Unless otherwise specified, the terms "bright" and "dim" refer to the epoch among the two where the observed spectral flux is larger and smaller and do not necessarily imply an underlying physical mechanism.

${ }^{\mathrm{b}}$ The sample average of the redshift.

${ }^{\mathrm{c}}$ The sample average of the magnitude difference, $\Delta M_{g}=M_{g}(\operatorname{dim})-M_{g}($ bright), between the dim and bright phases.

${ }^{\mathrm{d}}$ The uncertainty in the sample-averaged magnitude difference, by propagating the uncertainty in spectral flux density to that in magnitude, and then to the uncertainty of the sample-averaged magnitude difference.

eThe $1 \sigma$ sample scatter in the $\Delta M_{g}$.

TABLE 7

BEST-FIT CHARACTERISTIC VARIABILITY TIME SCALE.

\begin{tabular}{lccccc}
\hline type & zero-point $F_{\text {var }}{ }^{\mathrm{a}}$ & $\tau_{s}(\text { year })^{\mathrm{b}}$ & asymptotic $F_{\text {var }}\left(\Delta \tau_{\text {rest }} \rightarrow \infty\right)$ & reduced $\chi^{2}$ & range in $\Delta \tau_{\text {rest }}($ day $)$ \\
\hline QSO & $0.012 \pm 0.003$ & $0.4 \pm 1.0$ & $0.022 \pm 0.002$ & 0.04 & $0-300$ \\
star & $0.010 \pm 0.069$ & $11.2 \pm 6.3$ & $0.035 \pm 0.035$ & 3.41 & $0-710$ \\
galaxy & $0.009 \pm 0.162$ & $8.5 \pm 1.6$ & $0.025 \pm 0.081$ & 19.52 & $0-500$ \\
\hline
\end{tabular}

${ }^{\text {a }} F_{\text {var }}$ at zero time lag.

${ }^{\mathrm{b}}$ Characteristic variability time scale in the restframe.

TABLE 8

BEST-Fit CHARACTERISTIC VARIABILITY TIME SCALE, AFTER QUADTRATURE SUBTRACTION OF VARIABILITY AT ZERO TIME LAG.

\begin{tabular}{lcccc}
\hline type & $\tau_{s}(\text { year })^{\mathrm{a}}$ & asymptotic $F_{\text {var }}\left(\Delta \tau_{\text {rest }} \rightarrow \infty\right)$ & reduced $\chi^{2}$ & range in $\Delta \tau_{\text {rest }}$ (day) \\
\hline QSO & $0.4 \pm 0.0$ & $0.018 \pm 0.005$ & 13.10 & $0-300$ \\
star & $0.8 \pm 0.0$ & $0.012 \pm 0.003$ & 49.71 & $0-710$ \\
galaxy & $1.4 \pm 0.0$ & $0.011 \pm 0.018$ & 501.97 & $0-500$ \\
\hline
\end{tabular}

${ }^{a}$ Characteristic variability time scale in the restframe. 
TABLE 9

BPT LINE-RATio DIFFERENCE BETWEen DIM AND BRIGHT PHASES, FOR VARIABLE CANDIDATES.

\begin{tabular}{lcccc}
\hline type & $\left\langle\Delta \log _{10}([\mathrm{~N} \mathrm{II}] / \mathrm{H} \alpha)\right\rangle^{\mathrm{a}}$ & $\left\langle\Delta \log _{10}([\mathrm{O} \mathrm{III}] / \mathrm{H} \beta)\right\rangle$ & $\sigma\left(\Delta \log _{10}([\mathrm{~N} \mathrm{II}] / \mathrm{H} \alpha)\right)^{\mathrm{b}}$ & $\sigma\left(\Delta \log _{10}([\mathrm{O} \mathrm{III}] / \mathrm{H} \beta)\right)$ \\
\hline Seyfert 2 & $0.02 \pm 0.03$ & $0.06 \pm 0.08$ & 0.03 & 0.03 \\
Starforming & $0.09 \pm 0.01$ & $0.16 \pm 0.03$ & 0.17 & 0.21 \\
Composite & $0.03 \pm 0.03$ & $0.11 \pm 0.09$ & 0.03 & 0.08 \\
\hline eClass C & $0.17 \pm 0.05$ & $0.47 \pm 0.12$ & 0.18 & 0.43 \\
eClass D & $0.07 \pm 0.02$ & $0.15 \pm 0.06$ & 0.08 & 0.17 \\
\hline
\end{tabular}

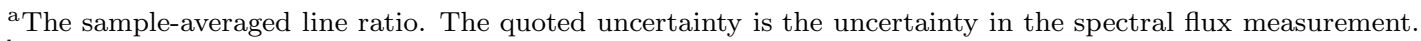

${ }^{\mathrm{b}}$ The $1 \sigma$ sample scatter in the line-ratio difference between dim and bright phases.

TABLE 10

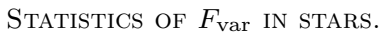

\begin{tabular}{lccc}
\hline method & mean & $\sigma_{m}$ & $\sigma$ \\
\hline No refinement & 0.035 & 0.0011 & 0.053 \\
SYS+BAND & 0.012 & 0.0003 & 0.013 \\
\hline
\end{tabular}

Note. - See caption in Table 2 for the meaning of the statistics. There are 2,379 common spectral pairs in each of the refinement approaches. The statistics of $F_{\mathrm{var}}$ are calculated at spectral $\mathrm{S} / \mathrm{N} \geq 10$.

TABLE 11

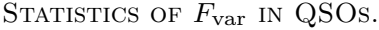

\begin{tabular}{lccc}
\hline method & mean & $\sigma_{m}$ & $\sigma$ \\
\hline No refinement & 0.036 & 0.0012 & 0.034 \\
SYS+BAND & 0.015 & 0.0005 & 0.014 \\
\hline
\end{tabular}

Note. - See caption in Table 2 for the meaning of the statistics. There are 766 common spectral pairs in each of the refinement approaches. The statistics of $F_{\text {var }}$ are calculated at spectral $\mathrm{S} / \mathrm{N} \geq 10$.

TABLE 12

MATChED OBJeCTS BETWEEN GCVSa AND OUR SAMPLES.

\begin{tabular}{|c|c|c|c|c|c|c|c|c|c|}
\hline $\mathrm{RA}^{\mathrm{b}}$ (degree) & $\mathrm{DEC}^{\mathrm{b}}$ (degree) & specClass $^{\mathrm{c}}$ & $\mathrm{S} / \mathrm{N}$ & $\Delta \tau^{\mathrm{d}}$ & difference $^{\mathrm{e}}$ in $g$ & difference in $r$ & difference in $i$ & $F_{\text {var }}$ & nSigma $^{f}$ \\
\hline 218.750940 & -0.768439 & 1 & 20.4 & 53 & $0.13 \pm 0.49$ & $-0.05 \pm 0.30$ & $-0.09 \pm 0.41$ & 0.017 & 1 \\
\hline 118.540000 & 42.817851 & 1 & 39.0 & 7 & $-0.02 \pm 0.26$ & $0.00 \pm 0.16$ & $0.00 \pm 0.21$ & 0.005 & 0 \\
\hline 119.352760 & 43.207637 & 1 & 43.0 & 7 & $0.05 \pm 0.23$ & $0.01 \pm 0.16$ & $-0.02 \pm 0.22$ & 0.011 & 3 \\
\hline 120.915900 & 42.512477 & 1 & 46.5 & 7 & $-0.23 \pm 0.20$ & $-0.03 \pm 0.13$ & $0.08 \pm 0.18$ & 0.051 & 4 \\
\hline 133.434070 & 57.811289 & 1 & 52.2 & 40 & $0.16 \pm 0.18$ & $0.06 \pm 0.12$ & $0.00 \pm 0.18$ & 0.045 & 4 \\
\hline
\end{tabular}

aThe General Catalogue of Variable Stars (Kholopov et al. 1999).

${ }^{\mathrm{b}}$ Given in J2000.

${ }^{\mathrm{c}}$ All of the matched objects are stars according to the SDSS spectral classification.

d The time lag $\Delta \tau$ is the difference between the two MJDs of observation, MJD(epoch 2)-MJD(epoch 1).

e The difference in magnitude between two epochs.

${ }^{f}$ The detection significance as a variable candidate, derived from this work. 
TABLE 13

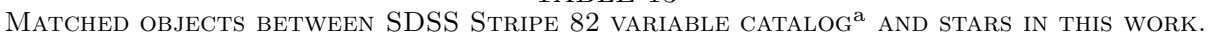

\begin{tabular}{|c|c|c|c|c|c|c|c|c|c|c|}
\hline 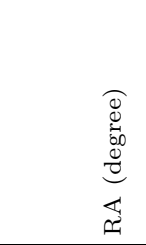 & 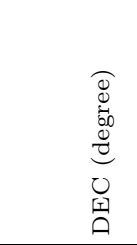 & 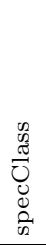 & $\mathrm{S} / \mathrm{N}$ & 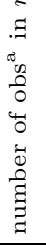 & $\Delta \tau$ & difference in $g$ & difference in $r$ & difference in $i$ & $F_{\text {var }}$ & nSigma \\
\hline 356.151320 & 0.303432 & 1 & 9.1 & 12 & 94 & $-0.01 \pm 2.13$ & $-0.02 \pm 0.68$ & $-0.04 \pm 0.73$ & 0.007 & 0 \\
\hline 356.171110 & -1.220269 & 1 & 26.8 & 10 & 94 & $0.08 \pm 0.53$ & $0.00 \pm 0.28$ & $-0.07 \pm 0.37$ & 0.006 & 0 \\
\hline 354.635580 & 0.856453 & 1 & 7.9 & 10 & 94 & $0.02 \pm 1.28$ & $-0.02 \pm 0.94$ & $-0.09 \pm 1.68$ & 0.039 & 1 \\
\hline 12.348661 & -0.299891 & 1 & 52.6 & 8 & 101 & $0.01 \pm 0.23$ & $0.00 \pm 0.15$ & $0.00 \pm 0.23$ & 0.000 & 0 \\
\hline 13.369355 & -0.722569 & 1 & 45.5 & 8 & 101 & $-0.13 \pm 0.35$ & $0.01 \pm 0.19$ & $0.05 \pm 0.27$ & 0.025 & 1 \\
\hline 31.675864 & 0.988745 & 1 & 17.0 & 14 & 65 & $-0.01 \pm 0.60$ & $0.00 \pm 0.35$ & $0.01 \pm 0.53$ & 0.001 & 0 \\
\hline 44.620576 & -0.609703 & 1 & 14.3 & 14 & 61 & $0.18 \pm 0.74$ & $0.01 \pm 0.45$ & $-0.09 \pm 0.71$ & 0.036 & 4 \\
\hline 44.322878 & 0.785284 & 1 & 13.2 & 14 & 61 & $-0.14 \pm 0.73$ & $-0.01 \pm 0.44$ & $0.06 \pm 0.68$ & 0.031 & 4 \\
\hline 46.057464 & -1.220639 & 1 & 29.6 & 12 & 56 & $-0.16 \pm 0.32$ & $-0.03 \pm 0.20$ & $0.07 \pm 0.29$ & 0.032 & 4 \\
\hline 48.387960 & 0.715234 & 1 & 17.0 & 14 & 108 & $-0.07 \pm 0.56$ & $-0.02 \pm 0.35$ & $0.03 \pm 0.58$ & 0.014 & 1 \\
\hline 49.984166 & -0.044137 & 1 & 8.6 & 14 & 108 & $-0.12 \pm 1.16$ & $-0.01 \pm 0.79$ & $0.05 \pm 1.39$ & 0.030 & 0 \\
\hline 51.883714 & 0.064299 & 1 & 54.1 & 12 & 32 & $-0.40 \pm 0.19$ & $-0.05 \pm 0.12$ & $0.15 \pm 0.17$ & 0.085 & 4 \\
\hline 52.295747 & 0.603985 & 1 & 20.9 & 12 & 32 & $-0.08 \pm 0.62$ & $-0.01 \pm 0.34$ & $0.04 \pm 0.49$ & 0.015 & 2 \\
\hline 52.476904 & 0.389118 & 1 & 45.5 & 14 & 32 & $0.02 \pm 0.28$ & $0.00 \pm 0.17$ & $0.00 \pm 0.24$ & 0.006 & 0 \\
\hline 51.602434 & 0.674580 & 1 & 45.2 & 14 & 20 & $0.01 \pm 0.24$ & $0.01 \pm 0.16$ & $-0.04 \pm 0.21$ & 0.007 & 4 \\
\hline 43.924804 & -0.542138 & 1 & 2.8 & 14 & 707 & $-0.10 \pm 4.41$ & $0.15 \pm 2.26$ & $0.17 \pm 3.21$ & 0.017 & 0 \\
\hline 44.201206 & 0.013831 & 1 & 19.2 & 14 & 707 & $-0.15 \pm 1.10$ & $-0.01 \pm 0.30$ & $0.03 \pm 0.30$ & 0.011 & 0 \\
\hline
\end{tabular}

Note. - See also captions in Table 12.

${ }^{a}$ Given by Sesar et al. (2007).

TABLE 14

Matched objects between SDSS Stripe 82 VARIAble CATAlog ${ }^{\text {a }}$ AND QSOS IN this work

\begin{tabular}{ccc}
\hline nSigma $^{\mathrm{b}}$ & number of objects & percentage \\
\hline 0 & 231 & 67.3 \\
1 & 45 & 13.1 \\
2 & 33 & 9.6 \\
3 & 10 & 2.9 \\
4 & 24 & 7.0 \\
\hline
\end{tabular}

${ }^{\mathrm{a}}$ Given by Sesar et al. (2007).

${ }^{\mathrm{b}}$ The detection significance as a variable candidate, derived from this work. 